DESY 96-189

hep-ph/9612435

December 1996

\title{
$O(\alpha)$ QED Corrections to Neutral Current Polarized Deep-Inelastic Lepton-Nucleon Scattering
}

\author{
Dmitri Bardin $^{a, b}$, Johannes Blümlein ${ }^{a}$, Pena Christova ${ }^{a, c}$, \\ and Lida Kalinovskaya ${ }^{a, b}$ \\ ${ }^{a} D E S Y-Z$ euthen, \\ Platanenallee 6, D-15735 Zeuthen, Germany \\ ${ }^{b}$ Laboratory for Theoretical Physics, JINR \\ ul. Joliot-Curie 6, RU-141980 Dubna, Russia \\ ${ }^{c}$ Dept. of Theoretical Physics, Faculty of Physics \\ Bishop Konstantin Preslavsky University, Shoumen, 9700, Bulgaria
}

\begin{abstract}
The $O(\alpha)$ leptonic QED corrections to neutral current polarized deep inelastic leptonnucleon scattering are calculated in leptonic variables both for the case of longitudinal and transverse nucleon polarization. The results of the complete calculation are compared with the corresponding leading log expressions. Numerical results are presented for the corrections in the kinematic range of the HERMES experiment and possible future polarized proton beam experiments at HERA.
\end{abstract}

PACS : 11.10.Gh,12.15.Lk,12.15.Mm,12.20.Ds,12.20.Fv,13.60.Fz. 


\section{Introduction}

Polarized deep-inelastic lepton-hadron scattering provides one of the cleanest methods to investigate the spin-structure of the nucleons. This field attracted much interest after the finding of the EMC experiment [1] in 1988 that the quarks appear to carry only a small fraction of the nucleon spin. During the last years precise measurements of both the polarized structure function $g_{1}\left(x, Q^{2}\right)$ of the proton and neutron by the SMC, SLAC, and HERMES experiments [2] were performed. Very recently also the structure function $g_{2}\left(x, Q^{2}\right)$ was measured for the first time [3].

The unfolding of the structure functions from the deep-inelastic scattering cross section requires the detailed knowledge of the electromagnetic radiative corrections, which are very large in some parts of the kinematic domain. A first calculation of the leptonic QED corrections for the case of pure photon exchange was performed in ref. [4] for the range of small values of $Q^{2}$ and a simple ansatz for the hadronic structure functions $g_{1}(x)$ and $g_{2}(x)$ [5].

In the present paper the leptonic $O(\alpha)$ QED corrections for the neutral current deep-inelastic scattering cross sections are calculated in a model independent approach for the case of longitudinal and transverse nucleon polarization. We account for both photon and $Z$ boson exchange. The resulting corrections are therefore applicable irrespectively of the $Q^{2}$ range. This is of particular importance for polarized deep-inelastic scattering experiments operating in the range of larger values of $Q^{2}$, e.g. possible future experiments in the kinematic regime being accessible at HERA, cf. [6], but also for the CERN fixed target experiments for the range of large $Q^{2}$.

The $O(\alpha)$ hadronic QED corrections are understood to be absorbed into the parton densities, as also the QCD corrections [0]-[10], leading to $Q^{2}$-dependent structure functions. In this way the hadronic tensor can be formulated quite generally in a model independent way. Particularly the structure functions need not necessarily to be represented using a partonic description.

Besides the complete $O(\alpha)$ results, we also derive the corrections in the leading logarithmic approximation (LLA). Due to their simpler analytical structure they are particularly suited for fast numerical estimates.

The paper is organized as follows. In section 2 the neutral current Born cross sections are derived. The leptonic $O(\alpha)$ QED corrections are presented in section 3. In section 4 we derive the different contributions to the $O(\alpha)$ QED corrections in the leading logarithmic approximation for the polarized case. Numerical results for the kinematic range of the HERMES experiment and possible future polarized ep collider experiments in the kinematic regime of HERA are presented in section 5. Section 6 contains the conclusions. An appendix summarizes kinematic relations used in the calculation.

\section{The Born Cross Section}

The Feynman diagram describing neutral current deep-inelastic lepton-nucleon scattering

$$
l\left(k_{1}\right)+p(p) \rightarrow l\left(k_{2}\right)+X\left(p^{\prime}\right)
$$

is shown in Figure 1. The particle 4-momenta are given in parentheses.

The matrix element for the Born cross section reads

$$
\mathcal{M}_{\text {Born }}=i e^{2}\left\langle p^{\prime}\left|\mathcal{J}_{\mu}\right| p\right\rangle \frac{1}{Q^{2}} \bar{u}\left(k_{2}\right)\left[Q_{l} \gamma^{\mu}+s_{l} \gamma^{\mu}\left(v_{l}+a_{l} \gamma_{5}\right) \chi\left(Q^{2}\right)\right] u\left(k_{1}\right) \frac{1}{(2 \pi)^{3}} \frac{1}{\sqrt{2 k_{1}^{0} 2 k_{2}^{0}}} .
$$


Here $e$ denotes the electromagnetic coupling, $q=k_{1}-k_{2}, m$ and $M$ are the lepton and nucleon masses, $\mathcal{J}_{\mu}$ is the hadronic current, $Q_{l}=s_{l}\left|Q_{l}\right|$ the charge of the electron, with $s_{l}=2 I_{l}^{(3)}$ twice the third component of the weak isospin of the electron, $v_{l}$ and $a_{l}$ are the vector and axial-vector couplings of the $Z$ boson to the electron

$$
v_{l}=1-4\left|Q_{l}\right| \sin ^{2} \theta_{W}, \quad a_{l}=1
$$

and $Q^{2}=-q^{2}$ denotes the four momentum transfer squared.

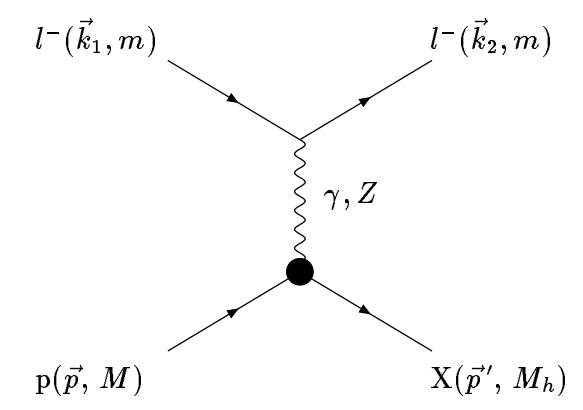

Figure 1: Born diagram for neutral current deep-inelastic lepton-proton scattering.

The calculation is performed in the on-mass-shell (OMS) scheme, in which the weak mixing angle $\theta_{W}$ is related to the weak boson masses by $\sin ^{2} \theta_{W}=1-M_{W}^{2} / M_{Z}^{2}$. Furthermore,

$$
\chi\left(Q^{2}\right)=\frac{G_{\mu}}{\sqrt{2}} \frac{M_{Z}^{2}}{8 \pi \alpha} \frac{Q^{2}}{Q^{2}+M_{Z}^{2}}
$$

is the $\gamma$ - $Z$ propagator ratio, with $G_{\mu}=1.16637 \cdot 10^{-5} \mathrm{GeV}^{-2}$ the Fermi constant, and $\alpha=1 / 137.06$ the fine structure constant.

The differential Born cross section is given by:

$$
d \sigma_{\text {Born }}=\frac{1}{j}(2 \pi)^{4} \sum_{\text {spins }}\left|\mathcal{M}_{\text {Born }}\right|^{2} d \vec{k}_{2} \prod_{i} d \vec{p}_{i}^{\prime} \delta^{4}\left(k_{1}+p-k_{2}-\sum_{i} p_{i}^{\prime}\right),
$$

where

$$
p^{\prime} \equiv \sum_{i} p_{i}^{\prime}=p+k_{1}-k_{2} \quad \text { and } \quad p^{\prime 2}=M_{h}^{2}
$$

$j$ denotes the flux factor

$$
j=\frac{\sqrt{\left(k_{1} \cdot p\right)^{2}-m^{2} M^{2}}}{k_{1}^{0} p^{0}} \frac{1}{(2 \pi)^{6}}=\frac{\sqrt{\lambda_{S}}}{2 k_{1}^{0} p^{0}(2 \pi)^{6}}
$$

with

$$
\lambda_{S}=S^{2}-4 m^{2} M^{2}, \quad S=\left(p+k_{1}\right)^{2}-m^{2}-M^{2} .
$$


For later use we rewrite the differential Born cross section (5) in terms of the leptonic and hadronic tensors, $L^{\mu \nu}$ and $W_{\mu \nu}$, integrating over the phase space up to two variables:

$$
d \sigma_{\text {Born }}=\frac{2 \alpha^{2}}{\sqrt{\lambda_{S}}} \frac{1}{Q^{4}}\left[L^{\mu \nu} W_{\mu \nu}\right] \frac{d \vec{k}_{2}}{k_{2}^{0}}=\frac{2 \pi \alpha^{2}}{\lambda_{S}} \frac{S^{2} y}{Q^{4}}\left[L^{\mu \nu} W_{\mu \nu}\right] d x d y .
$$

$x$ and $y$ are the Bjorken variables

$$
x=\frac{Q^{2}}{2 p \cdot q}, \quad y=\frac{p \cdot q}{p \cdot k_{1}} .
$$

The calculation is performed for incoming longitudinally polarized leptons. We used the spin density matrix

$$
\rho\left(k_{1}\right)=\sum_{s} u^{s}\left(k_{1}\right) \bar{u}^{s}\left(k_{1}\right)=\frac{1}{2}\left(1-\gamma_{5} \hat{\xi}_{l}\right)\left(\hat{k_{1}}+m\right) .
$$

The 4-vector of the lepton polarization is given by

$$
\xi_{l}=\frac{\lambda_{l}}{m}\left(k_{1}-\frac{2 m^{2}}{S} p\right) \frac{S}{\sqrt{\lambda_{S}}}
$$

with

$$
\xi_{l}^{2}=-\lambda_{l}^{2}, \quad \text { and } \quad \xi_{l} \cdot k_{1}=0 .
$$

$\lambda_{l}$ denotes the degree of lepton polarization. The leptonic tensor for neutral current polarized lepton (antilepton) scattering reads

$$
\begin{aligned}
L^{\mu \nu}= & {\left[2\left(k_{1}^{\mu} k_{2}^{\nu}+k_{1}^{\nu} k_{2}^{\mu}\right)-g^{\mu \nu} Q^{2}\right] L_{S}\left(Q^{2}, \lambda_{l}\right)-2 i k_{1 \alpha} k_{2 \beta} \varepsilon^{\alpha \beta \mu \nu} L_{A}\left(Q^{2}, \lambda_{l}\right) } \\
& +4 \frac{m^{2}}{S}\left\{i \varepsilon^{\alpha \beta \mu \nu}\left[p_{\alpha} q_{\beta} L_{v}\left(Q^{2}, \lambda_{l}\right)-p_{\alpha}\left(k_{1 \beta}+k_{2 \beta}\right) L_{a}\left(Q^{2}, \lambda_{l}\right)\right]\right. \\
& +\left[p^{\mu}\left(k_{1}^{\nu}+k_{2}^{\nu}\right)+p^{\nu}\left(k_{1}^{\mu}+k_{2}^{\mu}\right)-p^{\mu} q^{\nu}-p^{\nu} q^{\mu}\right. \\
& \left.\left.-g^{\mu \nu}\left[p \cdot\left(k_{1}+k_{2}\right)-p \cdot q\right]\right]\right\} L_{\chi}\left(Q^{2}, \lambda_{l}\right)+4 g^{\mu \nu} m^{2}\left[L_{\chi}\left(Q^{2}, \lambda_{l}\right)-L_{a}\left(Q^{2}, \lambda_{l}\right)\right] .
\end{aligned}
$$

The symmetric $(S)$ and antisymmetric $(A)$ parts are

$$
\begin{gathered}
L_{S}\left(Q^{2}, \lambda_{l}\right)=Q_{l}^{2}+2\left|Q_{l}\right|\left(v_{l}-p_{l} \lambda_{l} a_{l}\right) \chi\left(Q^{2}\right)+\left(v_{l}^{2}+a_{l}^{2}-2 p_{l} \lambda_{l} v_{l} a_{l}\right) \chi^{2}\left(Q^{2}\right), \\
L_{A}\left(Q^{2}, \lambda_{l}\right)=-\lambda_{l} Q_{l}^{2}+2\left|Q_{l}\right|\left(p_{l} a_{l}-\lambda_{l} v_{l}\right) \chi\left(Q^{2}\right)+\left(2 p_{l} v_{l} a_{l}-\lambda_{l}\left(v_{l}^{2}+a_{l}^{2}\right)\right) \chi^{2}\left(Q^{2}\right) .
\end{gathered}
$$

The formfactors contributing to the terms $\propto m^{2}$ are

$$
\begin{aligned}
& L_{v}\left(Q^{2}, \lambda_{l}\right)=\lambda_{l}\left[\left|Q_{l}\right|+v_{l} \chi\left(Q^{2}\right)\right]^{2}, \\
& L_{a}\left(Q^{2}, \lambda_{l}\right)=\lambda_{l} a_{l}^{2} \chi^{2}\left(Q^{2}\right), \\
& L_{\chi}\left(Q^{2}, \lambda_{l}\right)=\lambda_{l} p_{l} a_{l} \chi\left(Q^{2}\right)\left[\left|Q_{l}\right|+v_{l} \chi\left(Q^{2}\right)\right] .
\end{aligned}
$$


The particle label $p_{l}$ takes the values $p_{l}=1$ for particles and -1 for antiparticles.

The hadronic tensor reads

$$
W_{\mu \nu}=p^{0}(2 \pi)^{6} \sum \int\left\langle p^{\prime}\left|\mathcal{J}_{\mu}\right| p\right\rangle\left\langle p\left|\mathcal{J}_{\nu}\right| p^{\prime}\right\rangle \delta^{4}\left(p^{\prime}-\sum_{i} p_{i}^{\prime}\right) \prod_{i} d \vec{p}_{i}^{\prime}
$$

For its representation in terms of structure functions we follow the convention of ref. [11],

$$
\begin{aligned}
W_{\mu \nu}= & \left(-g_{\mu \nu}+\frac{q_{\mu} q_{\nu}}{q^{2}}\right) \mathcal{F}_{1}\left(x, Q^{2}\right)+\frac{\widehat{p_{\mu}} \widehat{p_{\nu}}}{p \cdot q} \mathcal{F}_{2}\left(x, Q^{2}\right)-i \varepsilon_{\mu \nu \lambda \sigma} \frac{q^{\lambda} p^{\sigma}}{2 p \cdot q} \mathcal{F}_{3}\left(x, Q^{2}\right) \\
& +i \varepsilon_{\mu \nu \lambda \sigma} \frac{q^{\lambda} s^{\sigma}}{p \cdot q} \mathcal{G}_{1}\left(x, Q^{2}\right)+i \varepsilon_{\mu \nu \lambda \sigma} \frac{q^{\lambda}\left(p \cdot q s^{\sigma}-s \cdot q p^{\sigma}\right)}{(p . q)^{2}} \mathcal{G}_{2}\left(x, Q^{2}\right) \\
& +\left[\frac{\widehat{p_{\mu}} \widehat{s_{\nu}}+\widehat{s_{\mu}} \widehat{p_{\nu}}}{2}-s . q \frac{\widehat{p_{\mu}} \widehat{p_{\nu}}}{p \cdot q}\right] \frac{1}{p \cdot q} \mathcal{G}_{3}\left(x, Q^{2}\right) \\
& +s . q \frac{\widehat{p_{\mu}} \widehat{p_{\nu}}}{(p . q)^{2}} \mathcal{G}_{4}\left(x, Q^{2}\right)+\left(-g_{\mu \nu}+\frac{q_{\mu} q_{\nu}}{q^{2}}\right) \frac{s . q}{p . q} \mathcal{G}_{5}\left(x, Q^{2}\right),
\end{aligned}
$$

where

$$
\widehat{p_{\mu}}=p_{\mu}-\frac{p \cdot q}{q^{2}} q_{\mu}, \quad \widehat{s_{\mu}}=s_{\mu}-\frac{s \cdot q}{q^{2}} q_{\mu}
$$

$s$ denotes the polarization 4 -vector of the nucleon. In the nucleon rest frame it is given by

$$
s=M\left(0, \vec{n}_{\lambda}\right)
$$

$\vec{n}_{\lambda}$ is an unit 3 -vector.

For a short-hand notation we have introduced the combined neutral current structure functions $\mathcal{F}_{i}$ and $\mathcal{G}_{i}$ in eq. ([19). In terms of the structure functions $F_{i}^{J_{1} J_{2}}$ and $g_{i}^{J_{1} J_{2}}$, which are associated with the respective currents, they read:

$$
\begin{aligned}
\mathcal{F}_{1,2}\left(x, Q^{2}\right)= & Q_{l}^{2} F_{1,2}^{\gamma \gamma}\left(x, Q^{2}\right)+2\left|Q_{l}^{2}\right|\left(v_{l}-p_{l} \lambda_{l} a_{l}\right) \chi\left(Q^{2}\right) F_{1,2}^{\gamma Z}\left(x, Q^{2}\right) \\
& +\left(v_{l}^{2}+a_{l}^{2}-2 p_{l} \lambda_{l} v_{l} a_{l}\right) \chi^{2}\left(Q^{2}\right) F_{1,2}^{Z Z}\left(x, Q^{2}\right) \\
\mathcal{F}_{3}\left(x, Q^{2}\right)= & 2\left|Q_{l}\right|\left(p_{l} a_{l}-\lambda_{l} v_{l}\right) \chi\left(Q^{2}\right) F_{3}^{\gamma Z}\left(x, Q^{2}\right) \\
& +\left[2 p_{l} v_{l} a_{l}-\lambda_{l}\left(v_{l}^{2}+a_{l}^{2}\right)\right] \chi^{2}\left(Q^{2}\right) F_{3}^{Z Z}\left(x, Q^{2}\right) \\
\mathcal{G}_{1,2}\left(x, Q^{2}\right)= & -Q_{l}^{2} p_{l} \lambda_{l} g_{1,2}^{\gamma \gamma}\left(x, Q^{2}\right)+2\left|Q_{l}\right|\left(p_{l} a_{l}-\lambda_{l} v_{l}\right) \chi\left(Q^{2}\right) g_{1,2}^{\gamma Z}\left(x, Q^{2}\right) \\
& +\left[2 p_{l} v_{l} a_{l}-\lambda_{l}\left(v_{l}^{2}+a_{l}^{2}\right)\right] \chi^{2}\left(Q^{2}\right) g_{1,2}^{Z Z}\left(x, Q^{2}\right) \\
\mathcal{G}_{3,4,5}\left(x, Q^{2}\right)= & 2\left|Q_{l}\right|\left(v_{l}-p_{l} \lambda_{l} a_{l}\right) \chi\left(Q^{2}\right) g_{3,4,5}^{\gamma Z}\left(x, Q^{2}\right) \\
& +\left(v_{l}^{2}+a_{l}^{2}-2 p_{l} \lambda_{l} v_{l} a_{l}\right) \chi^{2}\left(Q^{2}\right) g_{3,4,5}^{Z Z}\left(x, Q^{2}\right) .
\end{aligned}
$$


The twist-2 contributions to the structure functions can be expressed in terms of parton densities. In lowest order QCD one obtains [12, 13, 11] :

$$
\begin{aligned}
& F_{1}^{J_{1} J_{2}}\left(x, Q^{2}\right)=\sum_{q} \alpha_{J_{1} J_{2}}^{q}\left[q\left(x, Q^{2}\right)+\bar{q}\left(x, Q^{2}\right)\right], \\
& F_{2}^{J_{1} J_{2}}\left(x, Q^{2}\right)=2 x F_{1}^{J_{1} J_{2}}\left(x, Q^{2}\right), \\
& F_{3}^{J_{1} J_{2}}\left(x, Q^{2}\right)=\sum_{q} \beta_{J_{1} J_{2}}^{q}\left[q\left(x, Q^{2}\right)-\bar{q}\left(x, Q^{2}\right)\right], \\
& g_{1}^{J_{1} J_{2}}\left(x, Q^{2}\right)=\frac{1}{2} \sum_{q} \alpha_{J_{1} J_{2}}^{q}\left[\Delta q\left(x, Q^{2}\right)+\Delta \bar{q}\left(x, Q^{2}\right)\right], \\
& g_{2}^{J_{1} J_{2}}\left(x, Q^{2}\right)=-g_{1}^{J_{1} J_{2}}\left(x, Q^{2}\right)+\int_{x}^{1} \frac{d y}{y} g_{1}^{J_{1} J_{2}}\left(y, Q^{2}\right), \\
& g_{3}^{J_{1} J_{2}}\left(x, Q^{2}\right)=4 x \int_{x}^{1} \frac{d y}{y} g_{5}^{J_{1} J_{2}}\left(y, Q^{2}\right), \\
& g_{4}^{J_{1} J_{2}}\left(x, Q^{2}\right)=2 x g_{5}^{J_{1} J_{2}}\left(x, Q^{2}\right), \\
& g_{5}^{J_{1} J_{2}}\left(x, Q^{2}\right)=\sum_{q} \beta_{J_{1} J_{2}}^{q}\left[\Delta q\left(x, Q^{2}\right)-\Delta \bar{q}\left(x, Q^{2}\right)\right],
\end{aligned}
$$

where

$$
\begin{aligned}
\alpha_{J_{1} J_{2}}^{q} & =\alpha_{\gamma \gamma, \gamma Z, Z Z}^{q}=\left[\begin{array}{lll}
e_{q}^{2}, & 2 e_{q} v_{q}, & v_{q}^{2}+a_{q}^{2}
\end{array}\right], \\
\beta_{J_{1} J_{2}}^{q} & =\beta_{\gamma \gamma, \gamma Z, Z Z}^{q}=\left[\begin{array}{lll}
0, & 2 e_{q} a_{q}, & 2 v_{q} a_{q}
\end{array}\right],
\end{aligned}
$$

and the electroweak couplings are

$$
\begin{aligned}
e_{u} & =+\frac{2}{3}, & e_{d} & =-\frac{1}{3}, \\
v_{u} & =\frac{1}{2}-\frac{4}{3} \sin ^{2} \theta_{W}, & v_{d} & =-\frac{1}{2}+\frac{2}{3} \sin ^{2} \theta_{W}, \\
a_{u} & =\frac{1}{2}, & a_{d} & =-\frac{1}{2} .
\end{aligned}
$$

For the numerical results, which are presented in section 5 below, the structure functions eq. (29 33) are parametrized using a partonic description for the structure functions $F_{1}\left(x, Q^{2}\right), F_{3}\left(x, Q^{2}\right), g_{1}\left(x, Q^{2}\right)$ and $g_{5}\left(x, Q^{2}\right)$. The other structure functions are calculated using the relations above. $q\left(x, Q^{2}\right), \bar{q}\left(x, Q^{2}\right), \Delta q\left(x, Q^{2}\right)$, and $\Delta \bar{q}\left(x, Q^{2}\right)$ denote the unpolarized and polarized quark and antiquark densities, respectively.

It appears to be convenient to rewrite the Born cross section in terms of the following two contributions:

$$
\frac{d^{2} \sigma_{\text {Born }}}{d x d y}=\frac{d^{2} \sigma_{\text {Born }}^{\text {unpol }}}{d x d y}+\frac{d^{2} \sigma_{\text {Born }}^{\text {pol }}}{d x d y}
$$


with

$$
\frac{d^{2} \sigma_{\text {Born }}^{\text {unpol }}}{d x d y}=\frac{2 \pi \alpha^{2}}{Q^{4}} S \sum_{i=1}^{3} S_{i}^{U}(x, y) \mathcal{F}_{i}\left(x, Q^{2}\right),
$$

and

$$
\frac{d^{2} \sigma_{\text {Born }}^{\text {pol }}}{d x d y}=\frac{2 \pi \alpha^{2}}{Q^{4}} \lambda_{N}^{p} f^{p} S \sum_{i=1}^{5} S_{g i}^{p}(x, y) \mathcal{G}_{i}\left(x, Q^{2}\right) .
$$

$\lambda_{N}^{p}$ denotes the degree of nucleon polarization. For unpolarized deep-inelastic scattering only the first term, $d^{2} \sigma^{\text {unpol }}$, contributes. Eq. (39) applies both to the case of longitudinal $(L)$ and transversal $(T)$ nucleon polarization, where

$$
\begin{aligned}
f^{L} & =1, \\
f^{T} & =\cos \varphi \frac{d \varphi}{2 \pi} \sqrt{\frac{4 M^{2} x}{S y}\left(1-y-\frac{M^{2} x y}{S}\right)} \equiv \cos \varphi \frac{d \varphi}{2 \pi} \frac{1-y}{y} \sin \theta_{2} .
\end{aligned}
$$

$\theta_{2}$ is the angle between the incoming and outgoing leptons. $\varphi$ denotes the angle between the nucleon spin vector $s$ and the plane of the incoming and outgoing lepton in the nucleon rest frame (see figure 4 in appendix A). The polarization 3-vectors for longitudinal and transverse polarization are given by

$$
\begin{aligned}
\vec{n}^{L} & =\lambda_{N}^{L} \frac{\vec{k}_{1}}{\left|\vec{k}_{1}\right|}, \\
\vec{n}^{T} & =\lambda_{N}^{T} \vec{n}_{\perp}, \quad \text { with } \quad \vec{n}_{\perp} \vec{k}_{1}=0 .
\end{aligned}
$$

Finally, the kinematic coefficients in eqs. (38) and (39) are:

$$
\begin{gathered}
S_{1}^{U}(x, y)=2 x y^{2}, \\
S_{2}^{U}(x, y)=2\left[(1-y)-x y \frac{M^{2}}{S}\right], \\
S_{3}^{U}(x, y)=x\left[1-(1-y)^{2}\right], \\
S_{1}^{L}(x, y)=2 x y\left[(2-y)-2 \frac{M^{2}}{S} x y\right], \\
S_{2}^{L}(x, y)=-8 x^{2} y \frac{M^{2}}{S}, \\
S_{3}^{L}(x, y)=4 \frac{M^{2}}{S}\left[(1-y)-x y \frac{M^{2}}{S}\right], \\
S_{4}^{L}(x, y)=-2\left(1+\frac{2 x M^{2}}{S}\right)\left[(1-y)-x y \frac{M^{2}}{S}\right], \\
S_{5}^{L}(x, y)=-2 x y\left(y+2 \frac{M^{2}}{S} x y\right), \\
S_{1}^{T}\left(y, Q^{2}\right)=S_{5}^{T}\left(y, Q^{2}\right)=S_{1}^{U}\left(y, Q^{2}\right), \\
S_{2}^{T}\left(y, Q^{2}\right)=4 x y, \\
S_{3}^{T}\left(y, Q^{2}\right)=-\frac{S_{1}^{L}\left(y, Q^{2}\right)}{2 x y}, \\
S_{4}^{T}\left(y, Q^{2}\right)=S_{2}^{U}\left(y, Q^{2}\right) .
\end{gathered}
$$




\section{The $O(\alpha)$ Leptonic Correction}

The $O(\alpha)$ leptonic radiative corrections to the scattering cross sections are given by

$$
\frac{d^{2} \sigma_{\mathrm{rad}}^{\mathrm{QED}, 1}}{d x_{l} d y_{l}}=\frac{\alpha}{\pi} \delta_{\mathrm{VR}} \frac{d^{2} \sigma_{\mathrm{Born}}}{d x_{l} d y_{l}}+\frac{d^{2} \sigma_{\mathrm{Brems}}}{d x_{l} d y_{l}}=\frac{d^{2} \sigma_{\mathrm{rad}}^{\mathrm{unpol}}}{d x_{l} d y_{l}}+\frac{d^{2} \sigma_{\mathrm{rad}}^{\mathrm{pol}}}{d x_{l} d y_{l}} .
$$

The corrections are represented by the finite parts of the virtual and soft (VR) and Bremsstrahlung terms, respectively. Since we integrate over the phase space of the radiated photon, the differential cross sections (47) depend on the way in which the Bjorken variables $x$ and $y$ are determined kinematically. Because in all the polarized deep-inelastic scattering experiments performed so far the kinematic variables were measured using the scattered lepton the present calculation refers to this set of variables. The kinematic variables are defined by

$$
x_{l}=\frac{Q_{l}^{2}}{S y_{l}}, \quad y_{l}=\frac{p_{1} \cdot\left(k_{1}-k_{2}\right)}{p_{1} \cdot k_{1}}, \quad \text { and } \quad Q_{l}^{2}=\left(k_{1}-k_{2}\right)^{2} .
$$

The hadronic structure functions depend on the hadronic variables

$$
x_{h}=\frac{Q_{h}^{2}}{S y_{h}}, \quad y_{h}=\frac{p_{1} \cdot\left(p_{2}-p_{1}\right)}{p_{1} \cdot k_{1}}, \quad \text { and } \quad Q_{h}^{2}=\left(p_{2}-p_{1}\right)^{2}
$$

over which is integrated.

\subsection{Virtual and Soft Corrections}

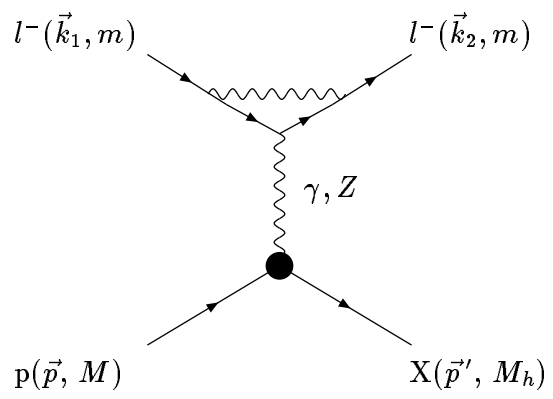

Figure 2: Diagram of the leptonic virtual photon correction for deep-inelastic scattering.

The virtual and soft correction, $\delta_{\mathrm{VR}}$, can be written as

$$
\begin{gathered}
\delta_{\mathrm{VR}}\left(y_{l}, Q_{l}^{2}\right)=\delta_{\mathrm{inf}}\left(y_{l}, Q_{l}^{2}\right)-\frac{1}{2} \ln ^{2}\left[\frac{1-y_{l}\left(1-x_{l}\right)}{1-y_{l} x_{l}}\right]+\operatorname{Li}_{2}\left[\frac{1-y_{l}}{\left(1-y_{l} x_{l}\right)\left(1-y_{l}\left(1-x_{l}\right)\right)}\right] \\
+\frac{3}{2} \ln \left(\frac{Q_{l}^{2}}{m^{2}}\right)-\operatorname{Li}_{2}(1)-2,
\end{gathered}
$$

\footnotetext{
${ }^{1}$ Other choices of kinematic variables were also investigated for the case of unpolarized deep-inelastic scattering $14,15,12,13$.
} 
with

$$
\delta_{\text {inf }}\left(y_{l}, Q_{l}^{2}\right)=\left[\ln \left(\frac{Q_{l}^{2}}{m^{2}}\right)-1\right] \ln \left[\frac{y_{l}^{2}\left(1-x_{l}\right)^{2}}{\left(1-y_{l} x_{l}\right)\left[1-y_{l}\left(1-x_{l}\right)\right]}\right]
$$

and

$$
\mathrm{Li}_{2}(x)=-\int_{0}^{1} d z \frac{\ln (1-x z)}{z} .
$$

These expressions are the same in the unpolarized and polarized case. They were derived in refs. [12, 13].

\subsection{Bremsstrahlung Corrections}

The differential Bremsstrahlung cross section for the scattering of polarized electrons off polarized protons, originating from the diagrams in figure 3 , is

$$
\frac{d \sigma_{\mathrm{Brem}}}{d x_{l} d y_{l}}=2 \alpha^{3} S y_{l} \int d y_{h} d Q_{h}^{2} \frac{1}{Q_{h}^{4}}\left[\frac{1}{2 \pi} \frac{d \varphi_{k}}{\sqrt{\lambda_{q}}} \frac{1}{4}\left(L_{\mathrm{rad}}^{\mu \nu} W_{\mu \nu}\right)\right] .
$$
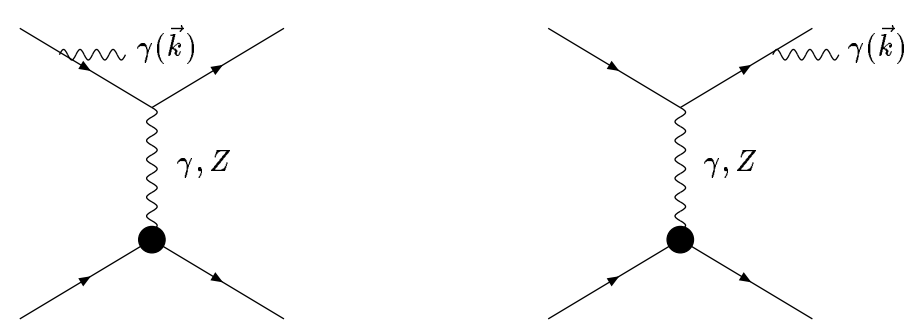

Figure 3: The leptonic Bremsstrahlung diagrams for for deep-inelastic scattering.

$W_{\mu \nu}$ denotes the hadronic tensor (19). The invariant $\lambda_{q}$ is defined in (A .14). The Bremsstrahlung correction to the leptonic tensor, $L_{\text {rad }}^{\mu \nu}$ has the following form :

$$
\begin{aligned}
L_{\mathrm{rad}}^{\mu \nu}= & 2 L_{S}\left(Q_{h}^{2}\right)\left\{4\left(-\frac{2 m^{2}}{z_{2}^{2}}+\frac{Q_{h}^{2}}{z_{1} z_{2}}\right) k_{1}^{\mu} k_{1}^{\nu}+4\left(-\frac{2 m^{2}}{z_{1}^{2}}+\frac{Q_{h}^{2}}{z_{1} z_{2}}\right) k_{2}^{\mu} k_{2}^{\nu}\right. \\
& \left.+\left[2 m^{2} Q_{h}^{2}\left(\frac{1}{z_{1}^{2}}+\frac{1}{z_{2}^{2}}\right)-\frac{Q_{l}^{4}+Q_{h}^{4}}{z_{1} z_{2}}-2\right] g_{\mu \nu}\right\} \\
& +4 i \varepsilon^{\alpha \beta \mu \nu}\left\{-L_{A}\left(Q_{h}^{2}, \lambda_{l}\right)\left[\left(\frac{2 m^{2}}{z_{2}^{2}}-\frac{Q_{l}^{2}}{z_{1} z_{2}}-\frac{1}{z_{2}}\right) k_{1 \alpha} q_{\beta}+\left(\frac{2 m^{2}}{z_{1}^{2}}-\frac{Q_{l}^{2}}{z_{1} z_{2}}+\frac{1}{z_{1}}\right) k_{2 \alpha} q_{\beta}\right]\right. \\
& +2\left[L_{v}\left(Q_{h}^{2}, \lambda_{l}\right)+L_{a}\left(Q_{h}^{2}, \lambda_{l}\right)\right] \frac{m^{2}}{z_{1}^{2}}\left[y_{l h} k_{1 \alpha} k_{2 \beta}+\left(1+y_{l h}\right) k_{2 \alpha} k_{\beta}\right] \\
& \left.-2\left[L_{v}\left(Q_{h}^{2}, \lambda_{l}\right)-L_{a}\left(Q_{h}^{2}, \lambda_{l}\right)\right] \frac{m^{2}}{z_{1}^{2}} k_{1 \alpha} k_{\beta}\right\} \\
& +8 L_{\chi}\left(Q_{h}^{2}, \lambda_{l}\right) \frac{m^{2}}{z_{1}^{2}}\left[2\left(k_{1}^{\mu} k_{2}^{\nu}+k_{1}^{\nu} k_{2}^{\mu}\right)-4\left(1+y_{l h}\right) k_{2}^{\mu} k_{2}^{\nu}-\left(Q_{l h}^{2}-y_{l h} Q_{h}^{2}\right) g^{\mu \nu}\right] .
\end{aligned}
$$


Here we retained only those terms in $O\left(\mathrm{~m}^{2}\right)$, which yield non-vanishing contributions after intgrating over $\varphi_{k}$ in the limit $m \rightarrow 0$. Similar to the leptonic tensor in the Born approximation, the tensor $L_{\text {rad }}^{\mu \nu}$ can be decomposed into a symmetric and an asymmetric term, and also a contribution $\propto m^{2}$. Besides the invariants defined in eqs. (48,49), it depends on

$$
z_{1}=2 k . k_{1}, \quad z_{2}=2 k . k_{2} .
$$

The finite part of the Bremsstrahlung contributions are expressed in form of integrals over the radiator functions $S_{X}^{i}\left(y_{l}, Q_{l}^{2}, y_{h}, Q_{h}^{2}\right)$ and $\mathcal{S}_{Y}^{i}\left(y_{l}, Q_{l}^{2}, y_{h}, Q_{h}^{2}\right)$. Since the structure of the differential cross sections turns out to be partly different for unpolarized and polarized deep-inelastic scattering, we will describe the individual cases separately.

\subsubsection{Unpolarized Case}

The finite contribution to the Bremsstrahlung cross section reads

$$
\begin{array}{r}
\frac{d \sigma_{\mathrm{Brem}}}{d x_{l} d y_{l}}=2 \alpha^{3} S \int_{\mathbf{R}} d y_{h} d Q_{h}^{2}\left\{\frac{1}{Q_{h}^{4}} \sum_{i=1}^{3} \mathcal{S}_{i}^{U}\left(y_{l}, Q_{l}^{2}, y_{h}, Q_{h}^{2}\right) \mathcal{F}_{i}\left(x_{h}, Q_{h}^{2}\right)\right. \\
\left.-\frac{1}{Q_{l}^{4}} \sum_{i=1}^{3} S_{i}^{U}\left(y_{l}, Q_{l}^{2}\right) \mathcal{L}^{\mathrm{IR}}\left(y_{l}, Q_{l}^{2}, y_{h}, Q_{h}^{2}\right) \mathcal{F}_{i}\left(x_{l}, Q_{l}^{2}\right)\right\}
\end{array}
$$

where

$$
\mathcal{L}^{\mathrm{IR}}\left(y_{l}, Q_{l}^{2}, y_{h}, Q_{h}^{2}\right)=\frac{Q_{l}^{2}+2 m^{2}}{Q_{l h}^{2}}\left(\frac{1}{\sqrt{C_{1}}}-\frac{1}{\sqrt{C_{2}}}\right)-m^{2}\left(\frac{B_{1}}{C_{1}^{3 / 2}}+\frac{B_{2}}{C_{2}^{3 / 2}}\right) .
$$

The integral over $\varphi_{k}$ in eq. (53) can be performed analytically, see appendix A. The remaining twofold integral takes the form [12]

$$
\int_{\mathbf{R}} d y_{h} d Q_{h}^{2}=\sum_{l=1}^{2} \int_{\hat{y}_{h_{l}}}^{\hat{y}_{h_{l+1}}} d y_{h} \int_{Q_{h_{1, d}}^{2}}^{Q_{h_{l, u}}^{2}} d Q_{h}^{2}
$$

with

$$
\begin{aligned}
\hat{y}_{h_{1}} & =\frac{y_{l}\left(y_{l} S-\sqrt{\lambda_{q}}\right)+2 Q_{l}^{2} M^{2} / S}{y_{l} S-\sqrt{\lambda_{q}}+M^{2}} \\
\hat{y}_{h_{2}} & =\frac{y_{l}\left(y_{l} S+\sqrt{\lambda_{q}}\right)+2 Q_{l}^{2} M^{2} / S}{y_{l} S+\sqrt{\lambda_{q}}+M^{2}} \\
\hat{y}_{h_{3}} & =y_{l} \\
Q_{h_{1, d}}^{2} & =Q_{l}^{2}+\frac{S}{2 M^{2}} y_{l h}\left(y_{l} S-\sqrt{\lambda_{q}}\right) \\
Q_{h_{2, u}}^{2} & =Q_{l}^{2}+\frac{S}{2 M^{2}} y_{l h}\left(y_{l} S+\sqrt{\lambda_{q}}\right) \\
Q_{h_{1, u}}^{2} & =y_{h} S,
\end{aligned}
$$

and

$$
y_{l h}=y_{l}-y_{h} .
$$


The functions $S_{i}^{U}\left(y_{l}, Q_{l}^{2}, y_{h}, Q_{h}^{2}\right)$ are given by:

$$
\begin{aligned}
S_{1}^{U}\left(y_{l}, Q_{l}^{2}, y_{h}, Q_{h}^{2}\right)= & y_{l}\left\{-2 m^{2} Q_{h}^{2}\left(\frac{B_{1}}{C_{1}^{3 / 2}}+\frac{B_{2}}{C_{2}^{3 / 2}}\right)\right. \\
& \left.+\frac{Q_{l}^{4}+Q_{h}^{4}}{Q_{l h}^{2}}\left(\frac{1}{\sqrt{C_{1}}}-\frac{1}{\sqrt{C_{2}}}\right)+\frac{2}{\sqrt{\lambda_{q}}}\right\}, \\
S_{2}^{U}\left(y_{l}, Q_{l}^{2}, y_{h}, Q_{h}^{2}\right)= & \frac{y_{l}}{S y_{h}}\left\{-2 m^{2}\left[\left(S^{2} y_{l_{1}}\left(y_{l_{1}}+y_{h}\right)-M^{2} Q_{h}^{2}\right) \frac{B_{1}}{C_{1}^{3 / 2}}\right.\right. \\
& \left.+\left(S^{2} y_{h_{1}}-M^{2} Q_{h}^{2}\right) \frac{B_{2}}{C_{2}{ }^{3 / 2}}\right] \\
& +\left[S^{2} Q_{h}^{2}\left(Y_{+}-y_{l} y_{h}\right)-M^{2}\left(Q_{l}^{4}+Q_{h}^{4}\right)\right] \frac{1}{Q_{l h}^{2}}\left(\frac{1}{\sqrt{C_{1}}}-\frac{1}{\sqrt{C_{2}}}\right) \\
& \left.-S^{2} y_{h}\left[\frac{1}{\sqrt{C_{1}}}+\frac{y_{l_{1}}}{\sqrt{C_{2}}}\right]-\frac{2 M^{2}}{\sqrt{\lambda_{q}}}\right\}, \\
S_{3}^{U}\left(y_{l}, Q_{l}^{2}, y_{h}, Q_{h}^{2}\right)= & \frac{y_{l}}{y_{h}}\left\{-m^{2} Q_{h}^{2}\left[\left(2 y_{l_{1}}+y_{h}\right) \frac{B_{1}}{C_{1}^{3 / 2}}+\left(2-y_{h}\right) \frac{B_{2}}{C_{2}^{3 / 2}}\right]\right. \\
& +\left(2-y_{l}\right) \frac{Q_{l}^{2} Q_{h}^{2}}{Q_{l h}^{2}}\left(\frac{1}{\sqrt{C_{1}}}-\frac{1}{\sqrt{C_{2}}}\right) \\
& \left.-Q_{h}^{2}\left(\frac{y_{l_{1}}}{\sqrt{C_{1}}}-\frac{1}{\sqrt{C_{2}}}\right)-\frac{y_{h}\left(Q_{l}^{2}+Q_{h}^{2}\right)}{2}\left(\frac{1}{\sqrt{C_{1}}}+\frac{1}{\sqrt{C_{2}}}\right)\right\},
\end{aligned}
$$

with

$$
\begin{aligned}
y_{l_{1}} & =1-y_{l}, \\
y_{h_{1}} & =1-y_{h}, \\
Q_{l h}^{2} & =Q_{l}^{2}-Q_{h}^{2} .
\end{aligned}
$$

The coefficients $B_{1,2}$ and $C_{1,2}$ are defined in eqs. (A.19 $A .22$. These functions were given in refs. 12, 13] before and are presented here in a somewhat more compact form.

\subsubsection{Longitudinal Polarization}

The Bremsstrahlung part of the differential cross section takes the form

$$
\begin{aligned}
\frac{d \sigma_{\text {Brem }}^{\text {Long }}}{d x_{l} d y_{l}}=2 \alpha^{3} & \lambda_{N}^{L} \int d y_{h} d Q_{h}^{2}\left\{\frac{1}{Q_{h}^{4}} \sum_{i=1}^{5} S_{i}^{L}\left(y_{l}, Q_{l}^{2}, y_{h}, Q_{h}^{2}\right) \mathcal{G}_{i}\left(x_{h}, Q_{h}^{2}\right)\right. \\
& -\frac{1}{Q_{l}^{4}} \sum_{i=1}^{5} S_{i}^{L}\left(y_{l}, Q_{l}^{2}\right) \mathcal{L}^{\mathrm{IR}}\left(y_{l}, Q_{l}^{2}, y_{h}, Q_{h}^{2}\right) \mathcal{G}_{i}\left(x_{h}, Q_{h}^{2}\right) \\
& +2 \frac{m^{2}}{Q_{h}^{4}} \frac{B_{1}}{C_{1}^{3 / 2}}\left[\sum_{i=1}^{2}\left(\mathcal{S}_{v i}^{L}\left(y_{l}, Q_{l}^{2}, y_{h}, Q_{h}^{2}\right) \mathcal{G}_{i}^{v}\left(x_{h}, Q_{h}^{2}\right)+\mathcal{S}_{a i}^{L}\left(y_{l}, Q_{l}^{2}, y_{h}, Q_{h}^{2}\right) \mathcal{G}_{i}^{a}\left(x_{h}, Q_{h}^{2}\right)\right)\right. \\
& \left.\left.+\sum_{i=3}^{5} \mathcal{S}_{\chi i}^{L}\left(y_{l}, Q_{l}^{2}, y_{h}, Q_{h}^{2}\right) \mathcal{G}_{i}^{\chi}\left(x_{h}, Q_{h}^{2}\right)\right]\right\} .
\end{aligned}
$$


As in the unpolarized case, to each of the structure functions corresponds a kinematic function $S_{i}^{L}\left(y_{l}, Q_{l}^{2}, y_{h}, Q_{h}^{2}\right)$. Furthermore, infrared-finite terms $\propto m^{2}$ contribute, which are denoted by $\mathcal{S}_{v i, a i}^{L}\left(y_{l}, Q_{l}^{2}, y_{h}, Q_{h}^{2}\right)$, and $\mathcal{S}_{\chi i}^{L}\left(y_{l}, Q_{l}^{2}, y_{h}, Q_{h}^{2}\right)$, respectively.

These coefficients are given by:

$$
\begin{aligned}
& S_{1}^{L}\left(y_{l}, Q_{l}^{2}, y_{h}, Q_{h}^{2}\right)=\frac{y_{l}}{S y_{h}}\left\{\left[S^{2}\left(2 y_{l_{1}}+y_{h}\right)-2 M^{2} Q_{l}^{2}\right] Q_{h}^{2}\right. \\
& \times\left[-2 m^{2} \frac{B_{1}}{C_{1}^{3 / 2}}+\frac{Q_{l}^{2}}{Q_{l h}^{2}}\left(\frac{1}{\sqrt{C_{1}}}-\frac{1}{\sqrt{C_{2}}}\right)\right] \\
& +\left[S^{2}\left(2-y_{h}\right)-2 M^{2} Q_{h}^{2}\right] Q_{h}^{2} \\
& \times\left[-2 m^{2} \frac{B_{2}}{C_{2}^{3 / 2}}+\frac{Q_{l}^{2}}{Q_{l h}^{2}}\left(\frac{1}{\sqrt{C_{1}}}-\frac{1}{\sqrt{C_{2}}}\right)\right] \\
& -\left[S^{2}\left(y_{h} Q_{l}^{2}+\left(2 y_{l_{1}}+y_{h}\right) Q_{h}^{2}\right)+2 M^{2} Q_{l}^{4}\right] \frac{1}{\sqrt{C_{1}}} \\
& -\left[S^{2}\left(y_{h} Q_{l}^{2}+\left(-2+y_{h}\right) Q_{h}^{2}\right)+2 M^{2} Q_{h}^{4}\right] \frac{1}{\sqrt{C_{2}}} \\
& \left.-\frac{4 M^{2}\left(Q_{l}^{2}+Q_{h}^{2}\right)}{\sqrt{\lambda_{q}}}\right\} \\
& S_{2}^{L}\left(y_{l}, Q_{l}^{2}, y_{h}, Q_{h}^{2}\right)=4 \frac{y_{l}}{S y_{h}^{2}} M^{2} Q_{h}^{2}\left\{( y _ { l _ { 1 } } + y _ { h } ) Q _ { l } ^ { 2 } \left[2 m^{2} \frac{B_{1}}{C_{1}^{3 / 2}}+\frac{1}{\sqrt{C_{1}}}\right.\right. \\
& \left.-\frac{Q_{l}^{2}}{Q_{l h}^{2}}\left(\frac{1}{\sqrt{C_{1}}}-\frac{1}{\sqrt{C_{2}}}\right)\right] \\
& +Q_{h}^{2}\left[2 m^{2} \frac{B_{2}}{C_{2}^{3 / 2}}-\frac{1}{\sqrt{C_{2}}}-\frac{Q_{l}^{2}}{Q_{l h}^{2}}\left(\frac{1}{\sqrt{C_{1}}}-\frac{1}{\sqrt{C_{2}}}\right)\right] \\
& \left.-Q_{l}^{2}\left(\frac{1}{\sqrt{C_{1}}}+\frac{y_{l_{1}}}{\sqrt{C_{2}}}\right)-\frac{y_{l}}{\sqrt{\lambda_{q}}}\right\} \\
& S_{3}^{L}\left(y_{l}, Q_{l}^{2}, y_{h}, Q_{h}^{2}\right)=\frac{y_{l}}{S^{2} y_{h}^{2}} M^{2}\left\{\left[S^{2}\left(y_{h} Q_{h}^{2}-\left(y_{l_{1}}+y_{h}\right)\left(2 y_{l_{1}}+y_{h}\right) Q_{l}^{2}\right)+2 M^{2} Q_{l}^{2} Q_{h}^{2}\right]\right. \\
& \times\left[2 m^{2} \frac{B_{1}}{C_{1}^{3 / 2}}-\frac{Q_{h}^{2}}{Q_{l h}^{2}}\left(\frac{1}{\sqrt{C_{1}}}-\frac{1}{\sqrt{C_{2}}}\right)\right] \\
& -2 Q_{h}^{2}\left(S^{2} y_{h_{1}}-M^{2} Q_{h}^{2}\right) \\
& \times\left[2 m^{2} \frac{B_{2}}{C_{2}{ }^{3 / 2}}-\frac{Q_{h}^{2}}{Q_{l h}^{2}}\left(\frac{1}{\sqrt{C_{1}}}-\frac{1}{\sqrt{C_{2}}}\right)\right] \\
& +2\left[S^{2}\left(-y_{h} Q_{l}^{2}+y_{h_{1}} Q_{h}^{2}\right)-M^{2}\left(Q_{l}^{4}+Q_{l}^{2} Q_{h}^{2}+Q_{h}^{4}\right)\right] \frac{1}{\sqrt{C_{1}}} \\
& +\left[S^{2}\left[y_{h} y_{h_{1}} Q_{l}^{2}+\left(2 y_{l_{1}}^{2}+y_{h}\right) Q_{h}^{2}\right]+2 M^{2} Q_{l}^{2} Q_{h}^{2}\right] \frac{1}{\sqrt{C_{2}}} \\
& \left.+\left[S^{2}\left(-4+y_{l}\right) y_{h}-4 M^{2}\left(Q_{h}^{2}+\frac{B_{2}}{\lambda_{q}}\right)\right] \frac{1}{\sqrt{\lambda_{q}}}\right\}
\end{aligned}
$$




$$
\begin{aligned}
& S_{4}^{L}\left(y_{l}, Q_{l}^{2}, y_{h}, Q_{h}^{2}\right)=\frac{y_{l}}{S^{2} y_{h}^{2}}\left\{\left(S^{2} y_{h}+2 M^{2} Q_{l}^{2}\right)\left[S^{2} y_{l_{1}}\left(y_{l_{1}}+y_{h}\right)-M^{2} Q_{h}^{2}\right]\right. \\
& \times\left[2 m^{2} \frac{B_{1}}{C_{1}{ }^{3 / 2}}-\frac{Q_{h}^{2}}{Q_{l h}^{2}}\left(\frac{1}{\sqrt{C_{1}}}-\frac{1}{\sqrt{C_{2}}}\right)\right] \\
& +\left(S^{2} y_{h}+2 M^{2} Q_{h}^{2}\right)\left[S^{2} y_{h_{1}}-M^{2} Q_{h}^{2}\right] \\
& \times\left[2 m^{2} \frac{B_{2}}{C_{2}^{3 / 2}}-\frac{Q_{h}^{2}}{Q_{l h}^{2}}\left(\frac{1}{\sqrt{C_{1}}}-\frac{1}{\sqrt{C_{2}}}\right)\right] \\
& +\left[\left(S^{2} y_{h}+2 M^{2} Q_{l}^{2}\right)\left[S^{2} y_{h}+M^{2}\left(Q_{l}^{2}+Q_{h}^{2}\right)\right]\right. \\
& \left.-2 M^{2} Q_{h}^{2}\left[S^{2} y_{h_{1}}-M^{2} Q_{h}^{2}\right]\right] \frac{1}{\sqrt{C_{1}}} \\
& +\left[\left(S^{2} y_{h}+2 M^{2} Q_{h}^{2}\right)\left[S^{2} y_{h} y_{l_{1}}-M^{2}\left(Q_{l}^{2}+Q_{h}^{2}\right)\right]\right. \\
& \left.-2 M^{2} Q_{h}^{2}\left[S^{2} y_{l_{1}}\left(y_{l_{1}}+y_{h}\right)-M^{2} Q_{h}^{2}\right]\right] \frac{1}{\sqrt{C_{2}}} \\
& \left.+2 M^{2}\left[S^{2}\left(2+y_{l_{1}}\right) y_{h}+2 M^{2}\left(Q_{h}^{2}+\frac{B_{2}}{\lambda_{q}}\right)\right] \frac{1}{\sqrt{\lambda_{q}}}\right\}, \\
& S_{5}^{L}\left(y_{l}, Q_{l}^{2}, y_{h}, Q_{h}^{2}\right)=\frac{y_{l}}{S y_{h}}\left\{\left(S^{2} y_{h}+2 M^{2} Q_{l}^{2}\right)\right. \\
& \times\left[2 m^{2} Q_{h}^{2} \frac{B_{1}}{C_{1}^{3 / 2}}-\frac{Q_{l}^{2} Q_{h}^{2}}{Q_{l h}^{2}}\left(\frac{1}{\sqrt{C_{1}}}-\frac{1}{\sqrt{C_{2}}}\right)-\frac{Q_{l}^{2}}{\sqrt{C_{1}}}\right] \\
& +\left(S^{2} y_{h}+2 M^{2} Q_{h}^{2}\right) \\
& \times\left[2 m^{2} Q_{h}^{2} \frac{B_{2}}{C_{2}{ }^{3 / 2}}-\frac{Q_{l}^{2} Q_{h}^{2}}{Q_{l h}^{2}}\left(\frac{1}{\sqrt{C_{1}}}-\frac{1}{\sqrt{C_{2}}}\right)-\frac{Q_{h}^{2}}{\sqrt{C_{2}}}-\frac{2}{\sqrt{\lambda_{q}}}\right] \\
& \left.+S^{2} y_{h}\left[\frac{Q_{h}^{2}}{\sqrt{C_{1}}}+\frac{Q_{l}^{2}}{\sqrt{C_{2}}}\right]-4 M^{2} \frac{B_{2}}{\lambda_{q}^{3 / 2}}\right\} \text {, }
\end{aligned}
$$

and

$$
\begin{aligned}
\mathcal{S}_{v 1}^{L}\left(y_{l}, Q_{l}^{2}, y_{h}, Q_{h}^{2}\right)= & -\frac{y_{l}}{S y_{h}}\left\{\left(S^{2} y_{l}+2 M^{2} Q_{l}^{2}\right)\left(Q_{l h}^{2}-y_{l h} Q_{h}^{2}\right)\right. \\
& \left.-S^{2} y_{l h}\left[Q_{l}^{2}-\left(1-y_{l h}\right) Q_{h}^{2}\right]\right\}, \\
\mathcal{S}_{a 1}^{L}\left(y_{l}, Q_{l}^{2}, y_{h}, Q_{h}^{2}\right)= & -\frac{y_{l}}{S y_{h}}\left\{\left(S^{2} y_{l}+2 M^{2} Q_{l}^{2}\right)\left(Q_{l h}^{2}-y_{l h} Q_{h}^{2}\right)\right. \\
& \left.+S^{2}\left[y_{l h}\left(Q_{l}^{2}+\left(1-y_{l h}\right) Q_{h}^{2}\right)-2 Q_{l h}^{2}\right]\right\}, \\
\mathcal{S}_{v 2}^{L}\left(y_{l}, Q_{l}^{2}, y_{h}, Q_{h}^{2}\right)= & -4 \frac{y_{l}}{S y_{h}^{2}} M^{2} Q_{l}^{2} y_{l h}^{2} Q_{h}^{2},
\end{aligned}
$$




$$
\begin{aligned}
\mathcal{S}_{a 2}^{L}\left(y_{l}, Q_{l}^{2}, y_{h}, Q_{h}^{2}\right)= & -4 \frac{y_{l}}{S y_{h}^{2}} M^{2} Q_{l}^{2}\left(Q_{l h}^{2}-y_{l h} Q_{l}^{2}+y_{l h}^{2} Q_{h}^{2}\right), \\
\mathcal{S}_{\chi 3}^{L}\left(y_{l}, Q_{l}^{2}, y_{h}, Q_{h}^{2}\right)= & \frac{y_{l}}{S^{2} y_{h}^{2}} M^{2}\left\{S^{2} Q_{l}^{2} y_{l h}^{2}\left[y_{l_{1}}+3\left(1-y_{l h}\right)\right]\right. \\
& \left.-\left(Q_{l h}^{2}-y_{l h} Q_{h}^{2}\right)\left[S^{2} y_{h}\left(2+\left(1-y_{l h}\right) \frac{Q_{l}^{2}}{Q_{h}^{2}}\right)+4 M^{2} Q_{l}^{2}\right]\right\}, \\
\mathcal{S}_{\chi 4}^{L}\left(y_{l}, Q_{l}^{2}, y_{h}, Q_{h}^{2}\right)= & \frac{y_{l}}{S^{2} y_{h}^{2}}\left(S^{2} y_{h}+2 M^{2} Q_{l}^{2}\right)\left\{-S^{2} y_{l h}^{2}\left(2 y_{l_{1}}+y_{h}\right)\right. \\
& \left.+\left(Q_{l h}^{2}-y_{l h} Q_{h}^{2}\right)\left[S^{2} y_{h}\left(1-y_{l h}\right) \frac{1}{Q_{h}^{2}}+2 M^{2}\right]\right\}, \\
\mathcal{S}_{\chi 5}^{L}\left(y_{l}, Q_{l}^{2}, y_{h}, Q_{h}^{2}\right)= & -2 \frac{y_{l}}{S y_{h}}\left(S^{2} y_{h}+2 M^{2} Q_{l}^{2}\right)\left(Q_{l h}^{2}-y_{l h} Q_{h}^{2}\right) .
\end{aligned}
$$

The combined structure functions $\mathcal{G}_{i}^{v, a, \chi}\left(x, Q^{2}\right) \operatorname{read}$

$$
\begin{aligned}
\mathcal{G}_{1,2}^{v}\left(x, Q^{2}\right) & =\lambda_{l}\left[Q_{e}^{2} g_{1,2}^{\gamma \gamma}\left(x, Q^{2}\right)+2\left|Q_{e}\right| v_{e} \chi\left(Q^{2}\right) g_{1,2}^{\gamma Z}\left(x, Q^{2}\right)+v_{e}^{2} \chi^{2}\left(Q^{2}\right) g_{1,2}^{Z Z}\left(x, Q^{2}\right)\right], \\
\mathcal{G}_{1,2}^{a}\left(x, Q^{2}\right) & =\lambda_{l} a_{e}^{2} \chi^{2}\left(Q^{2}\right) g_{1,2}^{Z Z}\left(x, Q^{2}\right), \\
\mathcal{G}_{3,4,5}^{\chi}\left(x, Q^{2}\right) & =\lambda_{l}\left[\left|Q_{e}\right| a_{e} \chi\left(Q^{2}\right) g_{3,4,5}^{\gamma Z}\left(x, Q^{2}\right)+v_{e} a_{e} \chi^{2}\left(Q^{2}\right) g_{3,4,5}^{Z Z}\left(x, Q^{2}\right)\right] .
\end{aligned}
$$

\subsubsection{Transverse Polarization}

Here the differential Bremsstrahlung contribution is given by

$$
\begin{aligned}
\frac{d \sigma_{\text {Brem }}^{\text {Trans }}}{d x_{l} d y_{l}}= & 2 \alpha^{3} \lambda_{N}^{T} \cos \varphi \frac{d \varphi}{2 \pi} \sqrt{\frac{4 M^{2} x_{l}}{S y_{l}}\left(1-y_{l}-\frac{M^{2} x_{l} y_{l}}{S}\right)} \\
\times & d d y_{h} d Q_{h}^{2}\left\{\frac{1}{Q_{h}^{4}} \sum_{i=1}^{5} S_{i}^{T}\left(y_{l}, Q_{l}^{2}, y_{h}, Q_{h}^{2}\right) \mathcal{G}_{i}\left(x_{h}, Q_{h}^{2}\right)\right. \\
& -\frac{1}{Q_{l}^{4}} \sum_{i=1}^{5} S_{i}^{T}\left(y_{l}, Q_{l}^{2}\right) \mathcal{L}^{\mathrm{IR}}\left(y_{l}, Q_{l}^{2}, y_{h}, Q_{h}^{2}\right) \mathcal{G}_{i}\left(x_{h}, Q_{h}^{2}\right) \\
& +2 \frac{m^{2}}{Q_{h}^{4}} \frac{1}{C_{1}{ }^{3 / 2}}\left[\sum_{i=1}^{2}\left(\mathcal{S}_{v i}^{T}\left(y_{l}, Q_{l}^{2}, y_{h}, Q_{h}^{2}\right) \mathcal{G}_{i}^{v}\left(x_{h}, Q_{h}^{2}\right)+\mathcal{S}_{a i}^{T}\left(y_{l}, Q_{l}^{2}, y_{h}, Q_{h}^{2}\right) \mathcal{G}_{i}^{a}\left(x_{h}, Q_{h}^{2}\right)\right)\right. \\
& \left.\left.+\sum_{i=3}^{5} \mathcal{S}_{\chi i}^{T}\left(y_{l}, Q_{l}^{2}, y_{h}, Q_{h}^{2}\right) \mathcal{G}_{i}^{\chi}\left(x_{h}, Q_{h}^{2}\right)\right]\right\} .
\end{aligned}
$$

The corresponding coefficients $S_{i}^{T}\left(y_{l}, Q_{l}^{2}, y_{h}, Q_{h}^{2}\right), \mathcal{S}_{v i, a i}^{T}\left(y_{l}, Q_{l}^{2}, y_{h}, Q_{h}^{2}\right)$, and $\mathcal{S}_{\chi i}^{T}\left(y_{l}, Q_{l}^{2}, y_{h}, Q_{h}^{2}\right)$, are :

$$
\begin{aligned}
S_{1}^{T}\left(y_{l}, Q_{l}^{2}, y_{h}, Q_{h}^{2}\right)= & S \frac{y_{l}^{2}}{y_{h}}\left\{\frac{S^{2} y_{l} y_{l h}+2 M^{2} Q_{l h}^{2}}{\lambda_{q}}\right. \\
& \times\left[-2 m^{2} Q_{h}^{2}\left(\frac{B_{1}}{C_{1}^{3 / 2}}-\frac{B_{2}}{C_{2}^{3 / 2}}\right)-\left(Q_{l}^{2}+Q_{h}^{2}\right)\left(\frac{1}{\sqrt{C_{1}}}+\frac{1}{\sqrt{C_{2}}}\right)\right] \\
& -2 m^{2} Q_{h}^{2}\left(\frac{B_{1}}{C_{1}^{3 / 2}}+\frac{B_{2}}{C_{2}{ }^{3 / 2}}\right)+Q_{h}^{2} \frac{Q_{l}^{2}+Q_{h}^{2}}{Q_{l h}^{2}}\left(\frac{1}{\sqrt{C_{1}}}-\frac{1}{\sqrt{C_{2}}}\right)
\end{aligned}
$$




$$
\begin{aligned}
& +Q_{l}^{2}\left(\frac{1}{\sqrt{C_{1}}}+\frac{1}{\sqrt{C_{2}}}\right) \\
& +2 \lambda_{k q h} \frac{S^{2} y_{l}+2 M^{2} Q_{l}^{2}}{\lambda_{q}} \\
& \times\left[2 m^{2} Q_{h}^{2}\left(\frac{1}{C_{1}^{3 / 2}}-\frac{1}{C_{2}^{3 / 2}}\right)\right. \\
& \left.\left.+\left(Q_{l}^{2}+Q_{h}^{2}\right)\left(\frac{1}{\sqrt{C_{1}}\left(B_{1}+\sqrt{\lambda_{q}} \sqrt{C_{1}}\right)}+\frac{1}{\sqrt{C_{2}}\left(B_{2}+\sqrt{\lambda_{q}} \sqrt{C_{2}}\right)}\right)\right]\right\}, \\
& S_{2}^{T}\left(y_{l}, Q_{l}^{2}, y_{h}, Q_{h}^{2}\right)=2 S \frac{y_{l}^{2}}{y_{h}^{2}}\left\{Q_{h}^{2}\left[1-y_{l h}-y_{l_{1}} \frac{S^{2} y_{l} y_{l h}+2 M^{2} Q_{l h}^{2}}{\lambda_{q}}\right]\right. \\
& \times\left[-2 m^{2} \frac{B_{1}}{C_{1}^{3 / 2}}+\frac{Q_{l}^{2}}{Q_{l h}^{2}}\left(\frac{1}{\sqrt{C_{1}}}-\frac{1}{\sqrt{C_{2}}}\right)-\frac{1}{\sqrt{C_{1}}}\right] \\
& +Q_{h}^{2}\left[1-\frac{S^{2} y_{l} y_{l h}+2 M^{2} Q_{l h}^{2}}{\lambda_{q}}\right] \\
& \times\left[-2 m^{2} \frac{B_{2}}{C_{2}^{3 / 2}}+\frac{Q_{l}^{2}}{Q_{l h}^{2}}\left(\frac{1}{\sqrt{C_{1}}}-\frac{1}{\sqrt{C_{2}}}\right)+\frac{1}{\sqrt{C_{2}}}\right] \\
& +2 \lambda_{k q h} Q_{h}^{2} \frac{S^{2} y_{l}+2 M^{2} Q_{l}^{2}}{\lambda_{q}} \\
& \times\left[-2 m^{2}\left(\frac{y_{l_{1}}}{C_{1}^{3 / 2}}+\frac{1}{C_{2}^{3 / 2}}\right)\right. \\
& +\frac{1}{\sqrt{C_{1}}\left(B_{1}+\sqrt{\lambda_{q}} \sqrt{C_{1}}\right)}-\frac{y_{l_{1}}}{\sqrt{C_{2}}\left(B_{2}+\sqrt{\lambda_{q}} \sqrt{C_{2}}\right)} \\
& \left.\left.+\frac{\left(2-y_{l}\right) Q_{h}^{2}}{Q_{l h}^{2}}\left(\frac{1}{\sqrt{C_{1}}\left(B_{1}+\sqrt{\lambda_{q}} \sqrt{C_{1}}\right)}-\frac{1}{\sqrt{C_{2}}\left(B_{2}+\sqrt{\lambda_{q}} \sqrt{C_{2}}\right)}\right)\right]\right\}, \\
& S_{3}^{T}\left(y_{l}, Q_{l}^{2}, y_{h}, Q_{h}^{2}\right)=\frac{y_{l}^{2}}{y_{h}^{2}}\left\{\frac{S^{2} y_{h} y_{l h}+2 M^{2} Q_{l h}^{2}}{\lambda_{q}}\right. \\
& \times\left[-m^{2}\left(\left[S^{2} y_{l_{1}}\left(2 y_{l_{1}}+y_{h}\right)-2 M^{2} Q_{h}^{2}\right] \frac{B_{1}}{C_{1}^{3 / 2}}\right.\right. \\
& \left.+\left[S^{2}\left(2-y_{h}\right)-2 M^{2} Q_{h}^{2}\right] \frac{B_{2}}{C_{2}^{3 / 2}}\right) \\
& +\left[S^{2} Q_{h}^{2}\left(Y_{+}-\frac{y_{l} y_{h}}{2}\right)-M^{2}\left(Q_{l}^{4}+Q_{h}^{4}\right)\right] \frac{1}{Q_{l h}^{2}}\left(\frac{1}{\sqrt{C_{1}}}-\frac{1}{\sqrt{C_{2}}}\right) \\
& \left.-\frac{S^{2} y_{h}}{2}\left(\frac{1}{\sqrt{C_{1}}}+\frac{y_{l_{1}}}{\sqrt{C_{2}}}\right)-\frac{2 M^{2}}{\sqrt{\lambda_{q}}}\right] \\
& +m^{2}\left(\left[S^{2}\left(1-y_{l h}\right)\left(2 y_{l_{1}}+y_{h}\right)-2 M^{2} Q_{h}^{2}\right] \frac{B_{1}}{C_{1}^{3 / 2}}\right. \\
& \left.+\left[S^{2}\left(2-y_{h}\right)-2 M^{2} Q_{h}^{2}\right] \frac{B_{2}}{C_{2}^{3 / 2}}\right)
\end{aligned}
$$




$$
\begin{aligned}
& -\left[S^{2} Q_{h}^{2}\left(Y_{+}+\frac{\left(3 y_{l_{1}}-y_{h_{1}}\right) y_{h}}{2}\right)-\frac{M^{2}\left(Q_{l}^{4}+Q_{h}^{4}\right)}{Q_{l h}^{2}}\left(\frac{1}{\sqrt{C_{1}}}-\frac{1}{\sqrt{C_{2}}}\right)\right] \\
& +\frac{S^{2} y_{h}}{2}\left(\frac{1}{\sqrt{C_{1}}}+\frac{1-y_{l h}}{\sqrt{C_{2}}}\right)+\frac{2 M^{2}}{\sqrt{\lambda_{q}}} \\
& +\lambda_{k q h} \frac{S^{2} y_{l}+2 M^{2} Q_{l}^{2}}{\lambda_{q}} \\
& {\left[2 m ^ { 2 } \left(\left[S^{2} y_{l_{1}}\left(2 y_{l_{1}}+y_{h}\right)-2 M^{2} Q_{h}^{2}\right] \frac{1}{C_{1}^{3 / 2}}\right.\right.} \\
& \left.+\left[S^{2}\left(2-y_{h}\right)-2 M^{2} Q_{h}^{2}\right] \frac{1}{C_{2}^{3 / 2}}\right) \\
& -\left[S^{2} Q_{h}^{2}\left(2 Y_{+}-y_{\iota} y_{h}\right)-2 M^{2}\left(Q_{l}^{4}+Q_{h}^{4}\right)\right] \\
& \times \frac{1}{Q_{l h}^{2}}\left(\frac{1}{\sqrt{C_{1}}\left(B_{1}+\sqrt{\lambda_{q}} \sqrt{C_{1}}\right)}-\frac{1}{\sqrt{C_{2}}\left(B_{2}+\sqrt{\lambda_{q}} \sqrt{C_{2}}\right)}\right) \\
& \left.\left.+S^{2} y_{h}\left(\frac{1}{\sqrt{C_{1}}\left(B_{1}+\sqrt{\lambda_{q}} \sqrt{C_{1}}\right)}+\frac{y_{l_{1}}}{\sqrt{C_{2}}\left(B_{2}+\sqrt{\lambda_{q}} \sqrt{C_{2}}\right)}\right)\right]\right\} \text {, } \\
& S_{4}^{T}\left(y_{l}, Q_{l}^{2}, y_{h}, Q_{h}^{2}\right)=\frac{y_{l}^{2}}{y_{h}^{2}}\left\{[ 1 - \frac { S ^ { 2 } y _ { l } y _ { l h } + 2 M ^ { 2 } Q _ { l h } ^ { 2 } } { \lambda _ { q } } ] \left[-2 m^{2}\left(\left[S^{2} y_{l_{1}}\left(1-y_{l h}\right)-M^{2} Q_{h}^{2}\right] \frac{B_{1}}{C_{1}^{3 / 2}}\right.\right.\right. \\
& \left.+\left[S^{2} y_{h_{1}}-M^{2} Q_{h}^{2}\right] \frac{B_{2}}{C_{2}{ }^{3 / 2}}\right) \\
& +\left[S^{2} Q_{h}^{2}\left(Y_{+}-y_{l} y_{h}\right)-M^{2}\left(Q_{l}^{4}+Q_{h}^{4}\right)\right] \frac{1}{Q_{l h}^{2}}\left(\frac{1}{\sqrt{C_{1}}}-\frac{1}{\sqrt{C_{2}}}\right) \\
& \left.-S^{2} y_{h}\left(\frac{1}{\sqrt{C_{1}}}+\frac{y_{l_{1}}}{\sqrt{C_{2}}}\right)-\frac{2 M^{2}}{\sqrt{\lambda_{q}}}\right] \\
& +2 \lambda_{k q h} \frac{S^{2} y_{l}+2 M^{2} Q_{l}^{2}}{\lambda_{q}} \\
& \times\left[-2 m^{2}\left(\left[S^{2} y_{l_{1}}\left(1-y_{l h}\right)-M^{2} Q_{h}^{2}\right] \frac{1}{C_{1}^{3 / 2}}\right.\right. \\
& \left.+\left[S^{2} y_{h_{1}}-M^{2} Q_{h}^{2}\right] \frac{1}{C_{2}{ }^{3 / 2}}\right) \\
& +\left[S^{2} Q_{h}^{2}\left(Y_{+}-y_{l} y_{h}\right)-M^{2}\left(Q_{l}^{4}+Q_{h}^{4}\right)\right] \\
& \times \frac{1}{Q_{l h}^{2}}\left(\frac{1}{\sqrt{C_{1}}\left(B_{1}+\sqrt{\lambda_{q}} \sqrt{C_{1}}\right)}-\frac{1}{\sqrt{C_{2}}\left(B_{2}+\sqrt{\lambda_{q}} \sqrt{C_{2}}\right)}\right) \\
& \left.\left.-S^{2} y_{h}\left(\frac{1}{\sqrt{C_{1}}\left(B_{1}+\sqrt{\lambda_{q}} \sqrt{C_{1}}\right)}+\frac{y_{l_{1}}}{\sqrt{C_{2}}\left(B_{2}+\sqrt{\lambda_{q}} \sqrt{C_{2}}\right)}\right)\right]\right\} \\
& S_{5}^{T}\left(y_{l}, Q_{l}^{2}, y_{h}, Q_{h}^{2}\right)=S \frac{y_{l}^{2}}{y_{h}}\left\{[ 1 - \frac { S ^ { 2 } y _ { l } y _ { l h } + 2 M ^ { 2 } Q _ { l h } ^ { 2 } } { \lambda _ { q } } ] \left[-2 m^{2} Q_{h}^{2}\left(\frac{B_{1}}{C_{1}^{3 / 2}}+\frac{B_{2}}{C_{2}^{3 / 2}}\right)\right.\right.
\end{aligned}
$$




$$
\begin{aligned}
& \left.+\frac{Q_{l}^{4}+Q_{h}^{4}}{Q_{l h}^{2}}\left(\frac{1}{\sqrt{C_{1}}}-\frac{1}{\sqrt{C_{2}}}\right)+\frac{2}{\sqrt{\lambda_{q}}}\right] \\
& +2 \lambda_{k q h} \frac{S^{2} y_{l}+2 M^{2} Q_{l}^{2}}{\lambda_{q}}\left[-2 m^{2} Q_{h}^{2}\left(\frac{1}{C_{1}{ }^{3 / 2}}+\frac{1}{C_{2}{ }^{3 / 2}}\right)\right. \\
& \left.\left.+\frac{Q_{l}^{4}+Q_{h}^{4}}{Q_{l h}^{2}}\left(\frac{1}{\sqrt{C_{1}}\left(B_{1}+\sqrt{\lambda_{q}} \sqrt{C_{1}}\right)}-\frac{1}{\sqrt{C_{2}}\left(B_{2}+\sqrt{\lambda_{q}} \sqrt{C_{2}}\right)}\right)\right]\right\}
\end{aligned}
$$

and

$$
\begin{aligned}
& \mathcal{S}_{v 1}^{T}\left(y_{l}, Q_{l}^{2}, y_{h}, Q_{h}^{2}\right)=S \frac{y_{l}^{2}}{y_{h}}\left[\left(Q_{l h}^{2}-y_{l h} Q_{h}^{2}\right) B_{1}-\left(Q_{l}^{2}+Q_{h}^{2}+y_{l h} Q_{h}^{2}\right) \mathcal{B}_{1}\right], \\
& \mathcal{S}_{a 1}^{T}\left(y_{l}, Q_{l}^{2}, y_{h}, Q_{h}^{2}\right)=S \frac{y_{l}^{2}}{y_{h}}\left(Q_{l h}^{2}-y_{l h} Q_{h}^{2}\right)\left(B_{1}+\mathcal{B}_{1}\right), \\
& \mathcal{S}_{v 2}^{T}\left(y_{l}, Q_{l}^{2}, y_{h}, Q_{h}^{2}\right)=2 S \frac{y_{l}^{2}}{y_{h}^{2}} Q_{h}^{2}\left[y_{l h}^{2} B_{1}-\left(y_{l}-y_{l_{1}} y_{l h}\right) \mathcal{B}_{1}\right], \\
& \mathcal{S}_{a 2}^{T}\left(y_{l}, Q_{l}^{2}, y_{h}, Q_{h}^{2}\right)=2 S \frac{y_{l}^{2}}{y_{h}^{2}}\left(Q_{l h}^{2}-y_{l h} Q_{h}^{2}\right)\left[\left(1-y_{l h}\right) B_{1}-y_{l_{1}} \mathcal{B}_{1}\right], \\
& \mathcal{S}_{\chi 3}^{T}\left(y_{l}, Q_{l}^{2}, y_{h}, Q_{h}^{2}\right)=\frac{y_{l}^{2}}{y_{h}^{2}} \frac{1}{2}\left\{\left[-S^{2} y_{l h}^{2}\left[y_{l_{1}}+3\left(1-y_{l h}\right)\right]\right.\right. \\
& \left.+\left(Q_{l h}^{2}-y_{l h} Q_{h}^{2}\right)\left(S^{2} y_{h} \frac{1-y_{l h}}{Q_{h}^{2}}+4 M^{2}\right)\right] B_{1} \\
& +\left[S^{2}\left(y_{l}\left(2-y_{l}\right)-\left(1+y_{l_{1}}^{2}\right) y_{l h}+3 y_{l_{1}} y_{l h}^{2}\right)\right. \\
& \left.\left.-\left(Q_{l h}^{2}-y_{l h} Q_{h}^{2}\right)\left(S^{2} y_{h} \frac{y_{l_{1}}}{Q_{h}^{2}}+4 M^{2}\right)\right] \mathcal{B}_{1}\right\}, \\
& \mathcal{S}_{\chi 4}^{T}\left(y_{l}, Q_{l}^{2}, y_{h}, Q_{h}^{2}\right)=\frac{y_{l}^{2}}{y_{h}^{2}}\left[S^{2} y_{l h}^{2}\left(2 y_{l_{1}}+y_{h}\right)\right. \\
& \left.-\left(Q_{l h}^{2}-y_{l h} Q_{h}^{2}\right)\left(S^{2} y_{h} \frac{1-y_{l h}}{Q_{h}^{2}}+2 M^{2}\right)\right]\left(B_{1}-\mathcal{B}_{1}\right), \\
& \mathcal{S}_{\chi 5}^{T}\left(y_{l}, Q_{l}^{2}, y_{h}, Q_{h}^{2}\right)=2 S \frac{y_{l}^{2}}{y_{h}}\left(Q_{l h}^{2}-y_{l h} Q_{h}^{2}\right)\left(B_{1}-\mathcal{B}_{1}\right) .
\end{aligned}
$$

The quantity $\mathcal{B}_{1}$, and similarly $\mathcal{B}_{2}$, which contain the denominator $\lambda_{q}$, may be simplified in the following way for the $O\left(\mathrm{~m}^{2}\right)$ terms,

$$
\begin{aligned}
\mathcal{B}_{1} & =B_{1} \frac{S^{2} y_{l} y_{l h}+2 M^{2} Q_{l h}^{2}}{\lambda_{q}}-2 \lambda_{k q h} \frac{S^{2} y_{l}+2 M^{2} Q_{l}^{2}}{\lambda_{q}} \\
& \equiv S^{2} y_{l h}\left(Q_{l h}^{2}-y_{l h} Q_{l}^{2}\right), \\
\mathcal{B}_{2} & =B_{2} \frac{S^{2} y_{l} y_{l h}+2 M^{2} Q_{l h}^{2}}{\lambda_{q}}-2 \lambda_{k q h} \frac{S^{2} y_{l}+2 M^{2} Q_{l}^{2}}{\lambda_{q}} \\
& \equiv S^{2} y_{l h} Q_{l h}^{2}\left(1+y_{h}\right)-S^{2} y_{l h}^{2} Q_{h}^{2}+2 M^{2}\left(Q_{l h}^{2}\right)^{2} .
\end{aligned}
$$


The explicit substitution of these terms in the above relations does not lead to a further simplification.

\section{The Leading Log Approximation}

In the leading logarithmic approximation the leptonic $O(\alpha)$ corrections to the differential scattering cross section can be written as

$$
\frac{d^{2} \sigma_{\mathrm{rad}}^{\mathrm{LLA}}}{d x d y}=\frac{d^{2} \sigma_{i}}{d x d y}+\frac{d^{2} \sigma_{f}}{d x d y}+\frac{d^{2} \sigma_{\mathrm{Comp}}}{d x d y}
$$

The individual contributions are due to the corresponding mass-singularities of initial-state $(i)$ and final-state $(f)$ radiation, and the Compton contribution, for which the mass singularity results from the low $Q^{2}$ range of the virtual photon in the sub-system Born term, in those situations which may be described partonically.

The initial and final-state radiation terms have the following structure:

$$
\frac{d^{2} \sigma_{i, f}^{k}}{d x d y}=\frac{\alpha}{2 \pi}\left(\ln \frac{Q^{2}}{m^{2}}-1\right) \int_{0}^{1} d z \frac{1+z^{2}}{1-z}\left\{\left.\theta\left(z-z_{0}\right) \mathcal{J} \frac{d^{2} \sigma_{\text {Born }}^{k}}{d x d y}\right|_{x=\hat{x}, y=\hat{y}, S=\hat{S}} ^{i, f}-\frac{d^{2} \sigma_{\text {Born }}^{k}}{d x d y}\right\}
$$

where the rescaled variables are

$$
\hat{S}=z S, \quad \hat{y}=\frac{y+z-1}{z}, \quad \hat{Q}^{2}=z Q^{2}, \quad \hat{x}=\frac{\hat{Q}^{2}}{\hat{y} \hat{S}}
$$

for initial-state radiation and

$$
\hat{S}=S, \quad \hat{y}=\frac{y+z-1}{z}, \quad \hat{Q}^{2}=\frac{Q^{2}}{z}, \quad \hat{x}=\frac{\hat{Q}^{2}}{\hat{y} \hat{S}},
$$

for final-state radiation. The Jacobian $\mathcal{J}$ is given by

$$
\mathcal{J} \equiv \mathcal{J}\left(x, y, Q^{2}\right)=\left|\frac{\partial(\hat{x}, \hat{y})}{\partial(x, y)}\right|
$$

cf. refs. [7, 16]- 19, 9]. The lower integration boundaries $z_{0}$ derive from the conditions

$$
\hat{x}\left(z_{0}\right) \leq 1, \quad \hat{y}\left(z_{0}\right) \leq 1
$$

and are given by

$$
z_{0}^{i}=\frac{1-y}{1-y x}, \quad z_{0}^{f}=1-y+x y
$$

The structure of eq. (94) is the same in the unpolarized and polarized case in leading order QED, since, as is well-known [20], the fermion-fermion splitting function

$$
P_{f f}(z)=\left(\frac{1+z^{2}}{1-z}\right)_{+}
$$


does not depend on the polarization of the incoming fermion in this order. Therefore, the Bremsstrahlung contributions for the polarized case are simply obtained in LLA by inserting in the relations refs. [0, 16]-19, 9] the differential Born cross section for the polarized case [21. Similar relations were also calculated in $O\left(\alpha^{2} \ln ^{2}\left(Q^{2} / m^{2}\right)\right)$ in refs. [22, 15].

For leptonic variables the Compton contributions depend on the type of nucleon polarization. These contributions are given by

$$
\begin{aligned}
\frac{d^{2} \sigma_{\mathrm{Comp}}^{U}}{d x_{l} d y_{l}} & =\frac{\alpha^{3}}{S x_{l}^{2}} \frac{Y_{+}}{y_{l_{1}}} \int_{x_{l}}^{1} d z \int_{\left(Q_{h}^{2}\right)^{\min }}^{\left(Q_{h}^{2}\right)^{\max }} \frac{d Q_{h}^{2}}{Q_{h}^{2}}\left[\frac{Z_{+}}{z} F_{2}^{\gamma \gamma}\left(x_{h}, Q_{h}^{2}\right)-z F_{L}^{\gamma \gamma}\left(x_{h}, Q_{h}^{2}\right)\right], \quad \text { cf. [17, 19, 16, 23], } \\
\frac{d^{2} \sigma_{\mathrm{Comp}}^{L}}{d x_{l} d y_{l}} & =\left(-2 \lambda_{l} \lambda_{N}^{L}\right) \frac{\alpha^{3}}{S x_{l}^{2}} \frac{Y_{-}}{y_{l_{1}}} \int_{x_{l}}^{1} d z \int_{\left(Q_{h}^{2}\right)^{\min }}^{\frac{\left(Q_{h}^{2}\right)^{\max }}{Q_{h}^{2}} \frac{Z_{-}}{z} x_{h} g_{1}^{\gamma \gamma}\left(x_{h}, Q_{h}^{2}\right)} \\
\frac{d^{2} \sigma_{\mathrm{Comp}}^{T}}{d x_{l} d y_{l}} & =\left(-2 \lambda_{l} \lambda_{N}^{T}\right) \frac{\alpha^{3}}{S x_{l}^{2}} \cos \varphi \frac{d \varphi}{2 \pi} \frac{y_{l}}{y_{l_{1}}^{2}} \sqrt{\frac{4 M^{2} x_{l}}{S y_{l}}\left(y_{l_{1}}-\frac{M^{2} x_{l} y_{l}}{S}\right)} \\
& \times \int_{x_{l}}^{1} \frac{d z}{z} \int_{\left(Q_{h}^{2}\right)^{\min }} \frac{d Q_{h}^{2}}{Q_{h}^{2}}\left\{\left(Y_{-}-y_{l} z\right) z x_{h} g_{1}^{\gamma \gamma}\left(x_{h}, Q_{h}^{2}\right)+2\left[Y_{+}(1-z)+y_{l_{1}}\right] x_{h} g_{2}^{\gamma \gamma}\left(x_{h}, Q_{h}^{2}\right)\right\} .
\end{aligned}
$$

Here we used the abbreviations

$$
\begin{gathered}
Y_{+}=1+\left(1-y_{l}\right)^{2}, \\
z=\frac{x_{l}}{x_{h}} .
\end{gathered}
$$

Part of the structure of eqs. (101) can be understood, as in the case of Bremsstrahlung, in terms of partonic splitting functions. In the unpolarized case

$$
P_{\gamma f}^{U}(z)=\frac{Z_{+}}{z}=\frac{1+(1-z)^{2}}{z}
$$

emerges [19, 9]. The longitudinal structure function is convoluted by the coefficient function

$$
c_{L}^{q}(z)=z
$$

cf. [24]. In the case of longitudinal nucleon polarization one has 20]

$$
P_{\gamma f}^{L}(z)=\frac{Z_{-}}{z}=\frac{1-(1-z)^{2}}{z}
$$

and the Compton term can as well be understood in the collinear parton model. Such an interpretation is, however, not possible in the case of transverse nucleon polarization, see ref. [11].

For the boundaries of the $Q_{h}^{2}$-integral in eqs. (101) we used

$$
\begin{aligned}
\left(Q_{h}^{2}\right)^{\max } & =\frac{x_{l}}{x_{h}}\left(1-y_{l}\right) Q_{l}^{2}, \\
\left(Q_{h}^{2}\right)^{\min } & =\max \left\{\left(Q_{h}^{2}\right)_{\mathrm{kin}}^{\min }, \bar{Q}_{h}^{2}, \hat{Q}_{h}^{2}\right\},
\end{aligned}
$$


where

$$
\begin{aligned}
\hat{Q}_{h}^{2} & =\left(\bar{M}_{h}^{2}-M^{2}\right) \frac{x_{h}}{1-x_{h}} \\
\left(Q_{h}^{2}\right)_{\mathrm{kin}}^{\min } & =\frac{x_{h}\left(x_{h} y_{l} S-Q_{l}^{2}\right)\left(y_{l} S-\sqrt{\lambda_{q}}\right)+2 Q_{l}^{2}\left(x_{h} M\right)^{2}}{2\left[x_{h} y_{l} S-Q_{l}^{2}+\left(x_{h} M\right)^{2}\right]} .
\end{aligned}
$$

$\bar{Q}_{h}^{2}$ and $\bar{M}_{h}^{2}$ denote respective cut values. The choosen value of $\left(Q_{h}^{2}\right)^{\text {max }}$ leads to a particularly good agreement of the LLA results and the complete $O(\alpha)$ corrections.

The LLA formulae are remarkably compact. The initial and final-state radiation contributions rely only on the respective Born cross sections in rescaled kinematic variables. Also the Compton terms exhibit a similarly simple structure. A numerical comparison of the complete leptonic $O(\alpha)$ corrections and the corresponding LLA results is given in section 5 .

\section{$5 \quad$ Numerical Results}

The QED corrections, which are discussed subsequently, are presented in terms of the correction factors

$$
\delta=\frac{d^{2} \sigma_{\mathrm{rad}}^{\mathrm{pol}}}{d^{2} \sigma_{\text {Born }}^{\mathrm{pol}}} \times 100 \%
$$

for the different nucleon polarizations and for different kinematic ranges. The calculations were performed with the code HECTOR [25]. For the parametrization of the polarized parton densities we use the 'standard' set (LO) of ref. [26]. The non-perturbative low $Q^{2}$-behavior of the polarized parton densities is modeled following an approach [27] which was used in ref. [13] in the unpolarized case before.

In Figure 5 the complete leptonic $O(\alpha)$ QED correction to the polarized part of the differential deep-inelastic scattering cross section for longitudinally polarized protons is shown for the kinematic range of the HERMES experiment at HERA at an electron beam energy of $E_{e}=27.5 \mathrm{GeV}$. Similarly to the case of unpolarized deep-inelastic scattering, see e.g. [12, 13], the corrections rise towards small $x$ and high $y$ values. The correction was calculated for the case of pure photon exchange. The structure functions were parametrized referring to the twist 2 contributions, using the parton densities of ref. [26]. The complete $O(\alpha)$ corrections are compared with the corresponding LLA results. A very good agreement of both descriptions is obtained for larger values of $x$ and lower and medium values of $y$. In the high $y$ range the LLA contributions yield somewhat larger corrections, which are further enhanced by the Compton contribution.

In Figure 6 the same corrections are considered applying a cut of $Q_{h}^{2}>1 \mathrm{GeV}^{2}$. The Compton part of the LLA contributions is widely removed by this condition. For smaller $x$ values the agreement between the complete corrections and the LLA contributions becomes somewhat worse.

Figure 7 shows the different contributions to the leptonic corrections in LLA for the kinematic regime of the HERMES experiment for longitudinal proton polarization. While the initial state corrections behave rising for growing $y$ and smaller values of $x$, the final state corrections are rather flat in $y$. The Compton contribution is only essential at small $x$ and large $y$.

In Figure 8 a comparison is given for the complete leptonic $O(\alpha)$ corrections and the corresponding LLA contributions for the polarized part of the differential scattering cross section 
in the case of transverse proton polarization for the kinematic range of the HERMES experiment. The $x$-dependence of the corrections is implied by the behavior of the structure functions $g_{1}\left(x, Q^{2}\right)$ and $g_{2}\left(x, Q^{2}\right)$. As in the case of longitudinally polarized protons, the complete and LLA corrections agree very well for small $y$ and larger values of $x$. In the high $y$ range both descriptions differ. Again the Compton term increases the difference slightly.

The effect of a cut of $Q_{h}^{2}>1 \mathrm{GeV}^{2}$ on the corrections for the case of transverse proton polarization is illustrated in Figure 9. The Compton contribution is removed by this cut as in the case of longitudinal proton polarization (Figure 6). For smaller values of $x$ the LLA terms deviate stronger from the complete corrections than in the case of longitudinal proton polarization.

In Figure 10 the different contributions to the LLA corrections are depicted for the case of transverse nucleon polarization. Similarly to the case of longitudinal nucleon polarization, the initial state radiation terms do widely determine the structure of the LLA correction. The final state radiation terms are flat in $y$ and the Compton terms contribute at small $x$ and large $y$ only.

The polarized contribution to the complete leptonic $O(\alpha)$ correction to the differential deepinelastic scattering cross section for longitudinally polarized protons in the kinematic range of the HERA collider at $\sqrt{S}=314 \mathrm{GeV}$ are shown in Figure 11. Their shape is rather similar to the corrections for lower values of $\sqrt{S}$, cf. Figure 5 . Lower values of $x, x \sim 10^{-3}$ can be probed. Here the corrections are rather large. The comparison with the LLA contributions is also shown. While the full LLA corrections agree rather well with the results of the complete calculation in the whole kinematic range, the contributions due to only initial and final state Bremsstrahlung differ in the high $y$ and low $x$ range from the complete $O(\alpha)$ corrections. The good agreement of the complete LLA relations is due to the choice of the Compton-logarithms, eq. (101, 107), which is particularly suited for larger values of $\sqrt{S}$.

In Figure 12 a comparison is shown of the polarized part of the complete leptonic $O(\alpha)$ QED corrections for longitudinally polarized protons at $\sqrt{S}=314 \mathrm{GeV}$ parametrizing the respective contributions to the polarized part of the scattering cross section by either only the structure function $g_{1}\left(x, Q^{2}\right)$ or the complete set of structure functions $g_{1}\left(x, Q^{2}\right), g_{4}\left(x, Q^{2}\right)$, and $g_{5}\left(x, Q^{2}\right)$, which are not suppressed kinematically by factors of $O\left(M^{2} / S\right)$, as the contributions due to the structure functions $g_{2}\left(x, Q^{2}\right)$ and $g_{3}\left(x, Q^{2}\right)$. Because of the different $y$ behavior of the kinematic factors weighting the structure functions, the correction changes significantly if only the structure function $g_{1}\left(x, Q^{2}\right)$ is considered in the scattering cross section. This applies also for the small $x$ and low $y$ range and is caused by the structure functions $g_{4}\left(x, Q^{2}\right)$ and $g_{5}\left(x, Q^{2}\right)$, which contribute to the $\gamma$ - $Z$ interference and $Z$-exchange contributions.

\section{Conclusions}

The $O(\alpha)$ leptonic QED corrections to the polarized differential neutral current deep-inelastic scattering cross sections were calculated both for the case of longitudinal and transverse nucleon polarization. This approach allows for a general description of structure functions, and is therefore particularly suited for experimental analyses, which aim on the unfolding of the different polarized structure functions from the measured cross sections. In the explicit calculations we considered a hadronic tensor which obeys Lorentz and time-reversal invariance, as well as current conservation. Its polarized part is described by the five structure functions $\left.g_{i}^{p}\left(x, Q^{2}\right)\right|_{i=1} ^{5}$. In the $O(\alpha)$ corrections the general structure of the Born cross section is resembled. The corrections can be expressed in terms of general kinematic factors and appropriate combinations of the 
neutral current structure functions, including both photon and $Z$-boson exchange. Additional combinations of structure functions contribute as well for terms $\propto m^{2}$. The latter terms yield finite contributions after integration in the limit $m \rightarrow 0$.

In the leading logarithmic approximation the $O(\alpha)$ leptonic QED corrections are described by the initial and final state Bremsstrahlung contributions and the Compton terms for pure photon exchange. While the structure of the Bremsstrahlung contributions is the same for the case of unpolarized and polarized deep-inelastic scattering, different expressions are obtained for the Compton terms. This is due to the behavior of the leading order QED splitting and coefficient functions in situations, in which the collinear parton model applies. The respective expressions are rather compact and their structure is partly induced by mass singularities, which allows an easy deduction of the corresponding expressions.

Numerically the $O(\alpha)$ corrections are rather large in the case of $e N$-scattering. Since the corrections behave $\sim \ln \left(Q^{2} / m^{2}\right)$, smaller corrections are obtained for the case of polarized $\mu N$ scattering. A numerical comparison with the corrections obtained by the code [5] was carried out in refs. [28]- 30] for the simplified assumptions in [5].

Both in the kinematic domain probed by the HERMES experiment and the kinematic range which would be accessible in possible future polarized deep inelastic scattering experiments in the HERA collider mode, the corrections grow towards small $x$ and high $y$. Except the range of large values of $y$ and small $x$ already the LLA results provide a very good description of the corrections. The structure of the corrections as a function of $x$ and $y$ is widely determined by the initial-state Bremsstrahlung terms. If cuts, e.g. on $Q_{h}^{2}$, are applied this agreement, however, becomes worse. While for longitudinal nucleon polarization the corrections are structurally similar to those in the unpolarized case, the corrections in the case of transverse nucleon polarization show a different $x$-behavior. 


\section{A Kinematic relations for the Bremsstrahlung process and an analytical integral}

We summarize a series of kinematic relations for the Bremsstrahlung process which are used in the present calculation. In the rest frame of the nucleon $(\vec{p}=0)$ the proton polarization 4 -vector $s$ and the lepton 4 -vectors are given by

$$
\begin{aligned}
s & =M(0, \cos \varphi, \sin \varphi, 0), \\
k_{1} & =\left(k_{10}, 0,0,\left|\vec{k}_{1}\right|\right), \\
k_{2} & =\left(k_{20},\left|\vec{k}_{2}\right| \sin \theta_{2}, 0,\left|\vec{k}_{2}\right| \cos \theta_{2}\right),
\end{aligned}
$$

with

$$
q_{l}=k_{1}-k_{2}=\left(k_{10}-k_{20},-\left|\vec{k}_{2}\right| \sin \theta_{2}, 0,\left|\vec{k}_{1}\right|-\left|\vec{k}_{2}\right| \cos \theta_{2}\right) .
$$

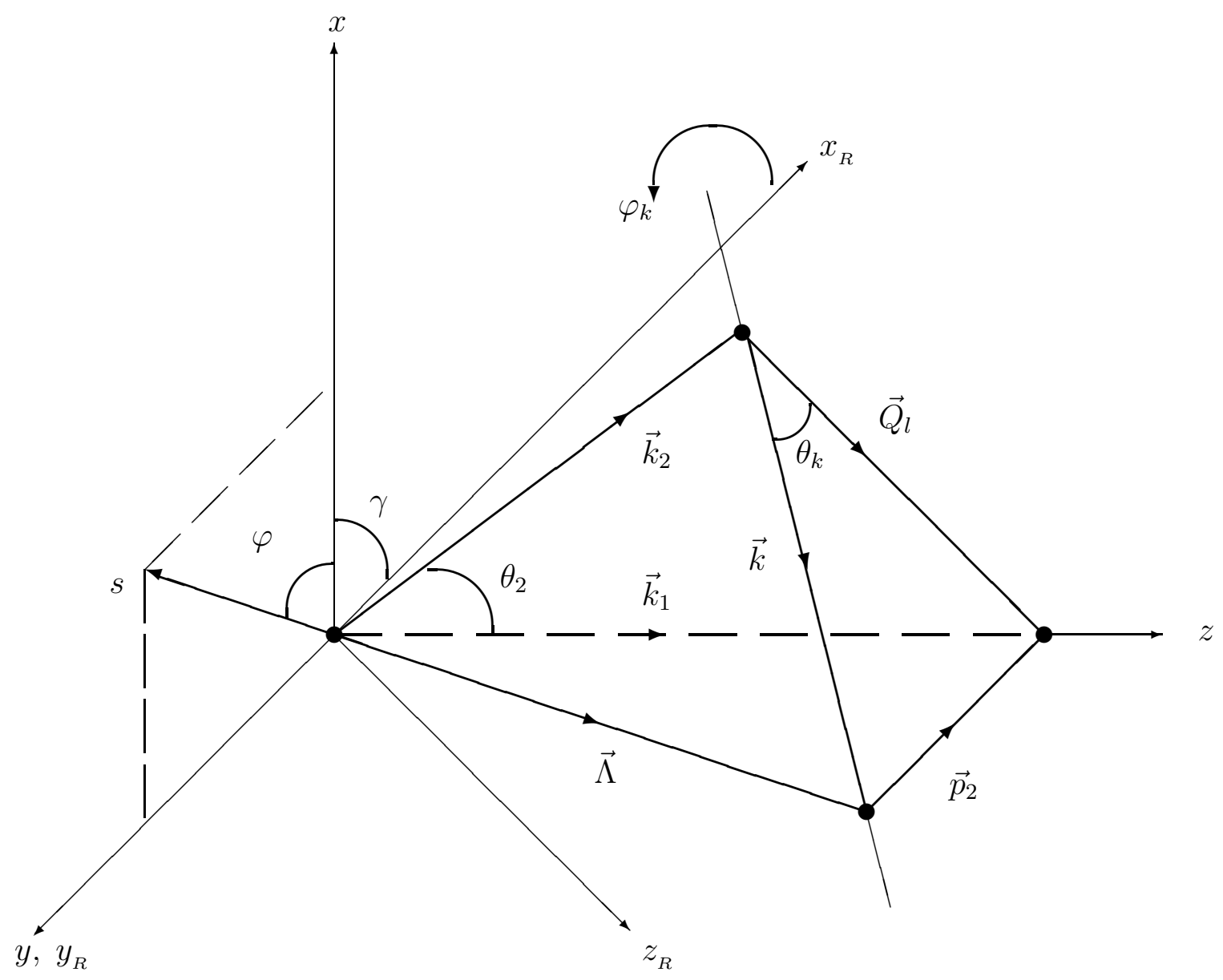

Figure 4: 3-vector relations for the Bremsstrahlung process in the rest frame of the nucleon.

We will also use a rotated frame in which two momentum components of $q$ vanish,

$$
\begin{aligned}
q_{l R} & ==\left(q_{l 0}, 0,0,\left|\vec{q}_{l}\right|\right) \\
k_{R} & =k_{0 R}\left(1, \sin \theta_{k} \cos \varphi_{k}, \sin \theta_{k} \sin \varphi_{k}, \cos \theta_{k}\right) .
\end{aligned}
$$


Here

$$
k_{0}=\frac{S y_{l h}}{2 M}, \quad k_{10}=\frac{S}{2 M}, \quad k_{20}=\frac{S y_{l_{1}}}{2 M}
$$

and

$$
|\vec{k}|=\frac{\sqrt{\lambda_{k}}}{2 M}, \quad\left|\vec{k}_{1}\right|=\frac{\sqrt{\lambda_{S}}}{2 M}, \quad\left|\vec{k}_{2}\right|=\frac{\sqrt{\lambda_{l}}}{2 M}
$$

The triangle functions $\lambda_{X}$ are

$$
\begin{aligned}
\lambda_{k} & =S^{2} y_{l h}^{2} \\
\lambda_{S} & =S^{2}-4 m^{2} M^{2}, \\
\lambda_{l} & =S^{2} y_{l_{1}}^{2}-4 m^{2} M^{2} .
\end{aligned}
$$

The sine and the cosine of the rotation angle $\gamma$ are

$$
\begin{aligned}
\cos \gamma & =\frac{\left|\vec{k}_{1}\right|-\left|\vec{k}_{2}\right| \cos \theta_{2}}{\left|\vec{Q}_{l}\right|}, \\
\sin \gamma & =\frac{\left|\vec{k}_{2}\right| \sin \theta_{2}}{\left|\vec{Q}_{l}\right|} .
\end{aligned}
$$

The transformation between the rest frame and the rotated frame for a general 4 -vector $K$ is given by

$$
\begin{aligned}
K_{0} & =K_{0 R}, \\
K_{x} & =K_{x R} \cos \gamma-K_{z R} \sin \gamma \\
K_{y} & =K_{y R}, \\
K_{z} & =K_{x R} \sin \gamma+K_{z R} \cos \gamma .
\end{aligned}
$$

The 4-vector $q$ is thus represented by

$$
q=\left(k_{10}-k_{20},-\left|\vec{k}_{2}\right| \sin \theta_{2}, 0,\left|\vec{k}_{1}\right|-\left|\vec{k}_{2}\right| \cos \theta_{2}\right)
$$

in the nucleon rest frame. The 4 -vector of the radiated photon is given by

$$
\begin{aligned}
& k_{0}=k_{0 R}, \\
& k_{x}=k_{0 R}\left(\sin \theta_{k} \cos \varphi_{k} \cos \gamma-\cos \theta_{k} \sin \gamma\right), \\
& k_{y}=k_{0 R} \sin \theta_{k} \sin \varphi_{k}, \\
& k_{z}=k_{0 R}\left(\sin \theta_{k} \cos \varphi_{k} \sin \gamma+\cos \theta_{k} \cos \gamma\right)
\end{aligned}
$$

The different angles defined in Figure 4 can be expressed in terms of the invariants (A.6, A .14) as follows

$$
\begin{aligned}
& \cos \theta_{2}=\frac{\lambda_{S}+\lambda_{l}-\lambda_{q}}{2 \sqrt{\lambda_{S}} \sqrt{\lambda_{l}}} \approx 1-\frac{2 M^{2} Q_{l}^{2}}{S^{2} y_{l_{1}}} \\
& \sin \theta_{2}=\frac{\sqrt{-\lambda\left(\lambda_{S}, \lambda_{l}, \lambda_{q}\right)}}{2 \sqrt{\lambda_{S}} \sqrt{\lambda_{l}}} \approx \frac{2 M^{2} \sqrt{\lambda_{S l q}}}{S^{2} y_{l_{1}}}
\end{aligned}
$$




$$
\begin{aligned}
\cos \gamma & =\frac{\lambda_{S}+\lambda_{q}-\lambda_{l}}{2 \sqrt{\lambda_{S}} \sqrt{\lambda_{q}}} \approx \frac{S^{2} y_{l}+2 M^{2} Q_{l}^{2}}{S \sqrt{\lambda_{q}}}, \\
\sin \gamma & =\frac{\sqrt{-\lambda\left(\lambda_{S}, \lambda_{l}, \lambda_{q}\right)}}{2 \sqrt{\lambda_{S}} \sqrt{\lambda_{q}}} \approx \frac{2 M^{2} \sqrt{\lambda_{S l q}}}{S \sqrt{\lambda_{q}}}, \\
\cos \theta_{k} & =\frac{\lambda_{k}+\lambda_{q}-\lambda_{h}}{2 \sqrt{\lambda_{k}} \sqrt{\lambda_{q}}} \approx \frac{S^{2} y_{l} y_{l h}+2 M^{2} Q_{l h}^{2}}{S y_{l h} \sqrt{\lambda_{q}}} \\
\sin \theta_{k} & =\frac{\sqrt{-\lambda\left(\lambda_{k}, \lambda_{l}, \lambda_{h}\right)}}{2 \sqrt{\lambda_{k}} \sqrt{\lambda_{q}}} \approx \frac{2 M^{2} \sqrt{\lambda_{k q h}}}{S y_{l h} \sqrt{\lambda_{q}}} .
\end{aligned}
$$

Here

$$
\lambda(x, y, z)=(x-y-z)^{2}-4 y z
$$

and

$$
\begin{aligned}
\lambda_{q} & =S^{2} y_{l}^{2}+4 M^{2} Q_{l}^{2}, \\
\lambda_{h} & =S^{2} y_{h}^{2}+4 M^{2} Q_{h}^{2}, \\
\lambda_{S l q} & =\frac{4 \lambda_{S} \lambda_{l}-\left(\lambda_{S}+\lambda_{l}-\lambda_{q}\right)^{2}}{16 M^{2}} \\
& \equiv S^{2} y_{l_{1}} Q_{l}^{2}-M^{2} Q_{l}^{4}-m^{2} \lambda_{q}, \\
\lambda_{k q h} & =\frac{4 \lambda_{k} \lambda_{q}-\left(\lambda_{q}+\lambda_{k}-\lambda_{h}\right)^{2}}{16 M^{2}} \\
& \equiv S^{2} y_{l h} d_{l h}-M^{2}\left(Q_{l h}^{2}\right)^{2},
\end{aligned}
$$

with

$$
d_{l h}=y_{l} Q_{h}^{2}-y_{h} Q_{l}^{2} .
$$

The latter terms in eqs. (A .12) result from the ultrarelativitic approximations in the lepton momenta. Furthermore, one obtains

$$
\begin{aligned}
\cos \gamma \cos \theta_{2}-\sin \gamma \sin \theta_{2} & =\frac{\lambda_{S}-\lambda_{l}-\lambda_{q}}{2 \sqrt{\lambda_{q}} \sqrt{\lambda_{l}}}, \\
\cos \gamma \sin \theta_{2}+\sin \theta_{\gamma} \sin \theta_{2} & =\frac{\sqrt{-\lambda\left(\lambda_{S}, \lambda_{l}, \lambda_{q}\right)}}{2 \sqrt{\lambda_{q}} \sqrt{\lambda_{l}}} .
\end{aligned}
$$

The integral over the angle $\varphi_{k}$ can be performed analytically. We express the phase space element as

$$
d \Gamma=\frac{\pi^{2} S^{3}}{4 \sqrt{\lambda_{S}}} y_{l} d x_{l} d y_{l} d y_{h} d Q_{h}^{2} \frac{1}{\pi} \frac{d z_{i}}{\sqrt{R_{i}}}=\frac{\pi^{2} S^{3}}{4 \sqrt{\lambda_{S}}} y_{l} d x_{l} d y_{l} d y_{h} d Q_{h}^{2} \frac{1}{\sqrt{\lambda_{q}}} \frac{1}{2 \pi} d \varphi_{k},
$$

with $i=1$ or 2 , and

$$
\begin{aligned}
& R_{i}=-\lambda_{q} z_{i}^{2}+2 B_{i} z_{i}-C_{i} \\
& B_{1}=S^{2}\left(Q_{l}^{2} y_{l_{1}} y_{l h}+d_{l h}\right)-2 M^{2} Q_{l}^{2} Q_{l h}^{2}, \\
& B_{2}=S^{2}\left(Q_{l}^{2} y_{l h}+y_{l_{1}} d_{l h}\right)+2 M^{2} Q_{l}^{2} Q_{l h}^{2}, \\
& C_{1}=S^{2}\left[\left(y_{l_{1}}+y_{h}\right) Q_{l}^{2}-Q_{h}^{2}\right]^{2}+4 m^{2}\left[S^{2} y_{l h} d_{l h}-M^{2}\left(Q_{l h}^{2}\right)^{2}\right], \\
& C_{2}=S^{2}\left[\left(1-y_{h}\right) Q_{l}^{2}-y_{l_{1}} Q_{h}^{2}\right]^{2}+4 m^{2}\left[S^{2} y_{l h} d_{l h}-M^{2}\left(Q_{l h}^{2}\right)^{2}\right] .
\end{aligned}
$$


These quantities are related by the identities

$$
\begin{aligned}
B_{1}+B_{2} & =\frac{C_{2}-C_{1}}{Q_{l h}^{2}}, \\
B_{2}-B_{1} & =\lambda_{q} Q_{l h}^{2} .
\end{aligned}
$$

With the help of the above relations the invariants $z_{1}$ and $z_{2}$, eq. (54), can be expressed as

$$
\begin{aligned}
& z_{1}=a_{1}-b \cos \varphi_{k}, \\
& z_{2}=a_{2}-b \cos \varphi_{k},
\end{aligned}
$$

where

$$
\begin{aligned}
a_{1} & =\frac{S \sqrt{\lambda_{k}}}{2 M^{2}}-\frac{\left(\lambda_{S}-\lambda_{q}-\lambda_{l}\right)\left(\lambda_{k}+\lambda_{q}-\lambda_{h}\right)}{8 M^{2} \lambda_{q}} \\
a_{2} & =\frac{S \sqrt{\lambda_{k}}}{2 M^{2}} y_{l_{1}}-\frac{\left(\lambda_{S}-\lambda_{q}-\lambda_{l}\right)\left(\lambda_{k}+\lambda_{q}-\lambda_{h}\right)}{8 M^{2} \lambda_{q}} \\
b & =\frac{\sqrt{-\lambda\left(\lambda_{S}, \lambda_{l}, \lambda_{q}\right)} \sqrt{-\lambda\left(\lambda_{k}, \lambda_{l}, \lambda_{h}\right)}}{8 M^{2} \lambda_{q}}
\end{aligned}
$$

The different $\varphi_{k}$-integrals

$$
[A]_{\varphi}=\frac{1}{2 \pi} \int_{0}^{2 \pi} \frac{d \varphi_{k}}{\sqrt{\lambda_{q}}} A\left(\varphi_{k}\right)
$$

are:

$$
\begin{aligned}
& {\left[z_{1}^{2}\right]_{\varphi}=\frac{3 B_{1(2)}^{2}-\lambda_{q} C_{1(2)}}{2 \lambda_{q}^{5 / 2}}} \\
& {\left[\frac{1}{z_{1(2)}^{2}}\right]_{\varphi}=\frac{B_{1(2)}}{C_{1(2)}^{3 / 2}}} \\
& {\left[\frac{1}{z_{1(2)}}\right]_{\varphi}=\frac{1}{C_{1(2)}^{1 / 2}}} \\
& {\left[\frac{1}{z_{1} z_{2}}\right]_{\varphi}=\frac{1}{Q_{l h}^{2}}\left[\frac{1}{\sqrt{C_{1}}}-\frac{1}{\sqrt{C_{2}}}\right]} \\
& {\left[z_{1(2)}\right]_{\varphi}=\frac{B_{1(2)}}{\lambda_{q}^{3 / 2}}} \\
& {[1]} \\
& {[1]_{\varphi}=\frac{1}{\sqrt{\lambda_{q}}}}
\end{aligned}
$$

$$
\begin{aligned}
& {\left[\frac{\cos \varphi_{k}}{z_{1(2)}}\right]_{\varphi}=\frac{b \lambda_{q}}{\sqrt{C_{1(2)}}\left(B_{1(2)}+\sqrt{\lambda_{q}} \sqrt{C_{1(2)}}\right)}} \\
& {\left[\frac{\cos \varphi_{k}}{z_{1(2)}^{2}}\right]_{\varphi}=\frac{b \lambda_{q}}{C_{1(2)}^{3 / 2}}} \\
& {\left[\frac{\cos \varphi_{k}}{z_{1} z_{2}}\right]_{\varphi}=\frac{b \lambda_{q}}{Q_{l h}^{2}}\left[\frac{1}{\sqrt{C_{1}}\left(B_{1}+\sqrt{\lambda_{q}} \sqrt{C_{1}}\right)}-\frac{1}{\sqrt{C_{2}}\left(B_{2}+\sqrt{\lambda_{q}} \sqrt{C_{2}}\right)}\right] .}
\end{aligned}
$$


Acknowledgement We would like to thank Prof. P. Söding for his constant support of the present project. P.C. and L.K. would like to thank DESY-Zeuthen for the hospitality extended to them. D.B. would like to thank I. Akushevich for useful discussions. We would like to thank P. Mulders for providing us ref. [3b]. The project was partly supported by EC-grant HCMPCT920004. and PECO-grant ERBCIPDCT-94-0016.

\section{References}

[1] EMC collaboration, J. Ashman et al., Phys. Lett. B206 (1988) 364; Nucl. Phys. B328 (1989) 1.

[2] for recent reviews see e.g.:

G. Altarelli and G. Ridolfi, Nucl. Phys. B (Proc. Suppl) 39BC (1995) 106;

G. Altarelli, CERN-TH-95/196;

J. Ellis and M. Karliner, in : Proc. of the Workshop on the Prospects of Spin Physics at HERA, DESY 95-200, eds. J. Blümlein and W.-D. Nowak, (DESY, Hamburg, 1995), p. 3; R. Voss, ibid. p. 25;

J. Feltesse and A. Schäfer, in: Proc. Future Physics at HERA, eds. G. Ingelman, A. De Roeck, and R. Klanner, Vol. 2 (DESY, Hamburg, 1996), 760;

G. Mallot, talk at the Int. Conference SPIN '96, Amsterdam.

[3] E143 Collaboration, K. Abe et al., Phys. Rev. Lett. 76 (1996) 587;

E154 Collaboration, T.D. Averett et al., Contribution to the Int. Conference SPIN '96, Amsterdam.

[4] I. Akushevich and T. Kukhto, Yad. Fiz. 52 (1990) 1442; Acta Phys. Polonica B22 (1991) 771 ;

I. Akushevich and N. Shumeiko, J. Phys. G20 (1994) 513;

I. Akushevich, A. Il'ichev, and N. Shumeiko, Yad. Fiz. 58 (1995) 2029.

[5] I. Akushevich and N. Shumeiko, in: Radiative Corrections for the HERMES Experiment, DESY-Zeuthen 94-02, eds. H. Böttcher and W.D. Nowak, p. 3;

I. Akushevich, N. Shumeiko, and A. Tolkachev, ibid. p. 43.

[6] J. Blümlein, in: [2c], p. 179 and DESY 95-164; hep-ph/9508387.

[7] A. De Rujula, R. Petronzio, and A. Savoy-Navarro, Nucl. Phys. B154 (1979) 394.

[8] J. Kripfganz and H. Perlt, Z. Phys. C41 (1988) 319.

[9] J. Blümlein, Z. Phys. C47 (1990) 89.

[10] H. Spiesberger, Phys. Rev. D52 (1995) 4936.

[11] J. Blümlein and N. Kochelev, Phys. Lett. B381 (1996) 296; DESY 96-175, hep-ph/9612318.

[12] A. Akhundov, D. Bardin, L. Kalinovskaya, and T. Riemann, Fortschr. Physik 44 (1996) 373. 
[13] A. Arbuzov, D. Bardin, J. Blümlein, L. Kalinovskaya, and T. Riemann, Comp. Phys. Commun. 94 (1996) 128, hep-ph/9511434.

[14] J. Blümlein, Phys. Lett. B271 (1991) 267.

[15] J. Blümlein, Z. Phys. C65 (1995) 293.

[16] M. Consoli and M. Greco, Nucl. Phys. B186 (1981) 519.

[17] W. Beenakker, F. Berends, and W. van Neerven, in : Proc. of the Workshop on Radiative Corrections for $e^{+} e^{-}$Collisions, Ringberg, 1989, ed. J.H. Kühn, (Springer, Berlin, 1989), p. 3.

[18] E. Kuraev, N. Merenkov, and V. Fadin, Sov. J. Nucl. Phys. 47 (1988) 1009.

[19] J. Blümlein, PHE 89-8 (June 1989), in : Proc. Int. Conf. Frontiers in Elementary Particle Physics, Kazimierz, Poland, May 1989, eds. Z. Ajduk, S. Pokorski, and A. Wroblewski, (World Scientific, Singapore, 1990), p. 274.

[20] D. Gross and F. Wilczek, Phys. Rev. D8 (1974) 416; D9 (1974) 980;

H. Georgi and D. Politzer, Phys. Rev. D9 (1974) 416;

K. Sasaki, Progr. Theor. Phys. 54 (1975) 1816;

M. Ahmed and G. Ross, Phys. Lett. B56 (1975) 385; Nucl. Phys. B111 (1976) 298;

G. Altarelli and G. Parisi, Nucl. Phys. B 126 (1977) 298.

[21] I. Akushevich and T. Kukhto, Yad. Fiz. 52 (1990) 1442.

[22] J. Kripfganz, H.-J. Möhring, and H. Spiesberger, Z. Phys. C 49 (1991) 501.

[23] J. Blümlein, G. Levman, and H. Spiesberger, in : Proc. of the 1990 Summer Study on High Energy Physics, Research Directions for the Decade, Snowmass, CO, ed. E. Berger, (World Scientific, Singapore, p. 554; J. Phys. G19 (1993) 1695.

[24] A. Zee, F. Wilczek, and S.B. Treiman, Phys. Rev. D10 (1974) 2881.

[25] HECTOR 1.11 User Manual, in preparation. The beta version of the code can be accessed through http://www.ifh.de/theory/publist.htm]

[26] M. Glück, E. Reya, M. Stratmann, and W. Vogelsang, Phys. Rev. D53 (1996) 4775.

[27] N. Volkonsky and L. Prokhorov, Sov. J. Exp. Theor. Phys. Letters 21 (1975) 389.

[28] D. Bardin, J. Blümlein, P. Christova and L. Kalinovskaya, in : Proc. of the 1996 HERA Physics Workshop, eds. G. Ingelman, R. Klanner, and A. De Roeck, (DESY, Hamburg, 1996), Vol. 2, p. 827, DESY 96-198, hep-ph/9609399.

[29] D. Bardin, Contribution to the Proceedings of the International Conference on High Energy Physics, Warsaw, August 1996.

[30] D. Bardin, J. Blümlein, P. Christova, L. Kalinovskaya, and T. Riemann, DESY 96-243, hep-ph/9611426, Acta Phys. Polonica B, in print. 


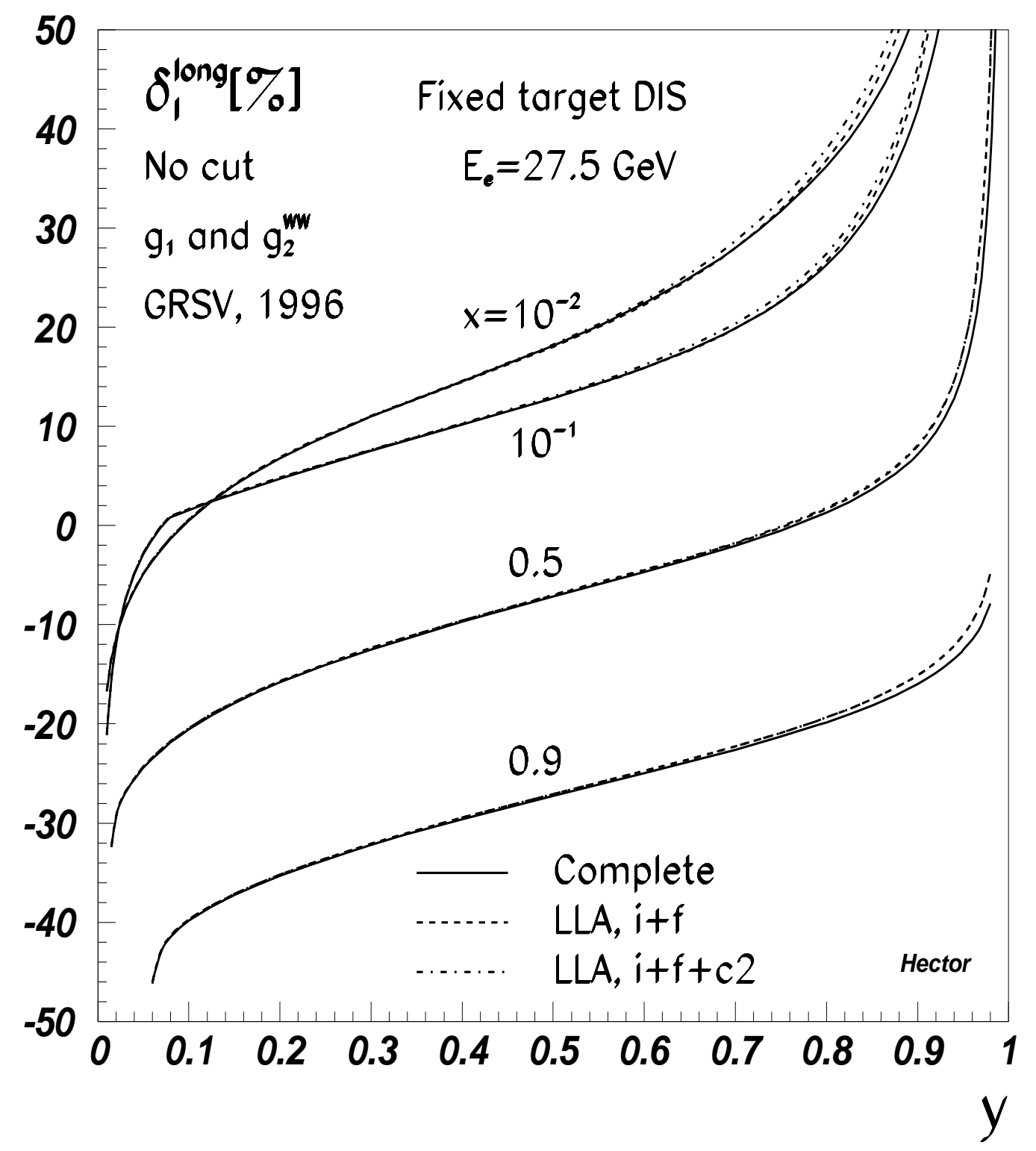

Figure 5: $O(\alpha)$ leptonic QED correction, eq. (47), to the polarized part of the differential deepinelastic scattering cross section for longitudinally polarized protons at $\sqrt{S}=7.4 \mathrm{GeV}$. Full lines: complete corrections; dashed lines : initial and final-state Bremsstrahlung contributions in LLA; dashdotted lines : complete LLA contributions, eq. (94). 


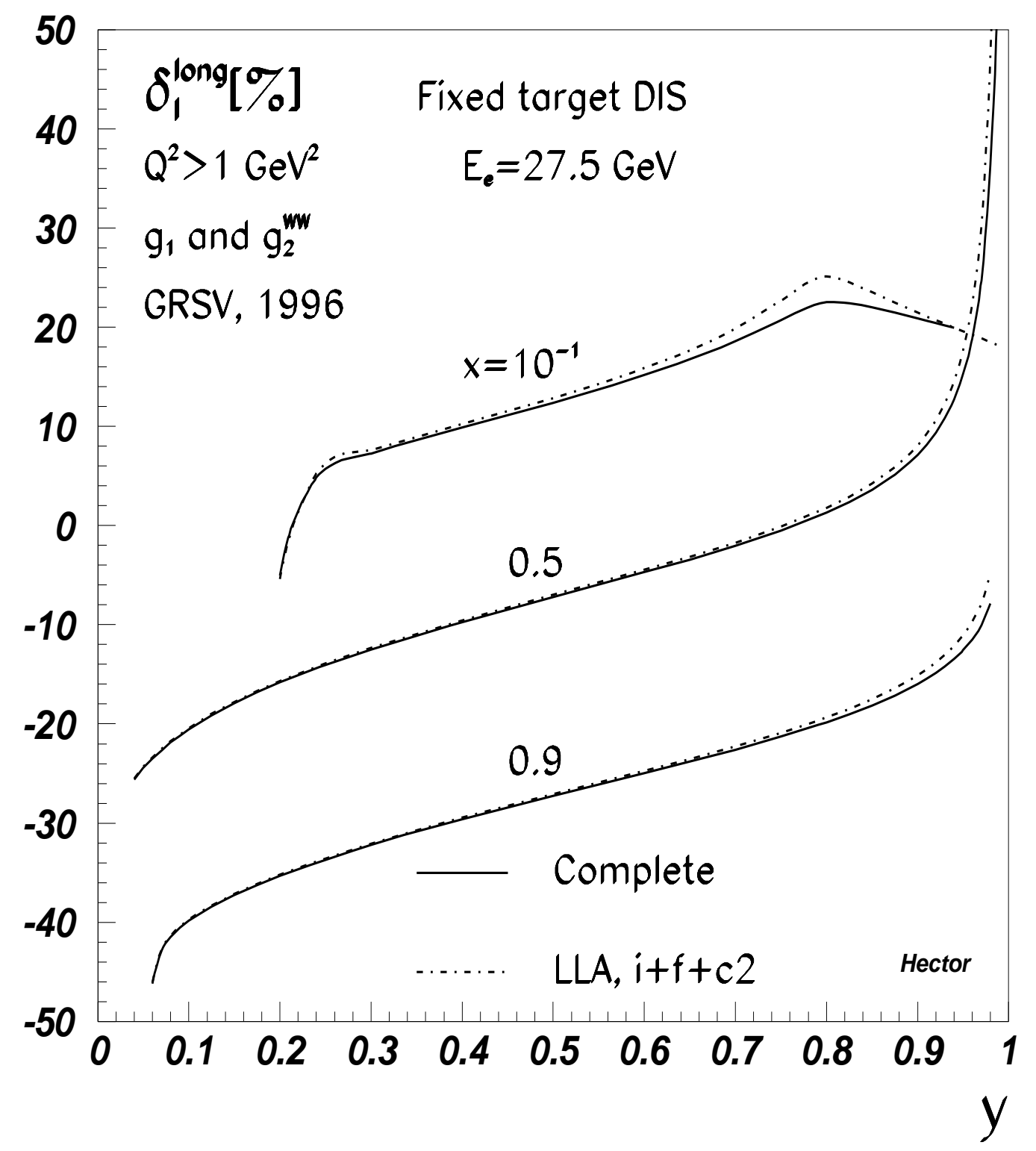

Figure 6 : The same as in figure 5, but for a $Q^{2}$-cut of $Q_{h}^{2}>1 \mathrm{GeV}$. Full lines : complete corrections; dash-dotted lines: complete LLA corrections. 


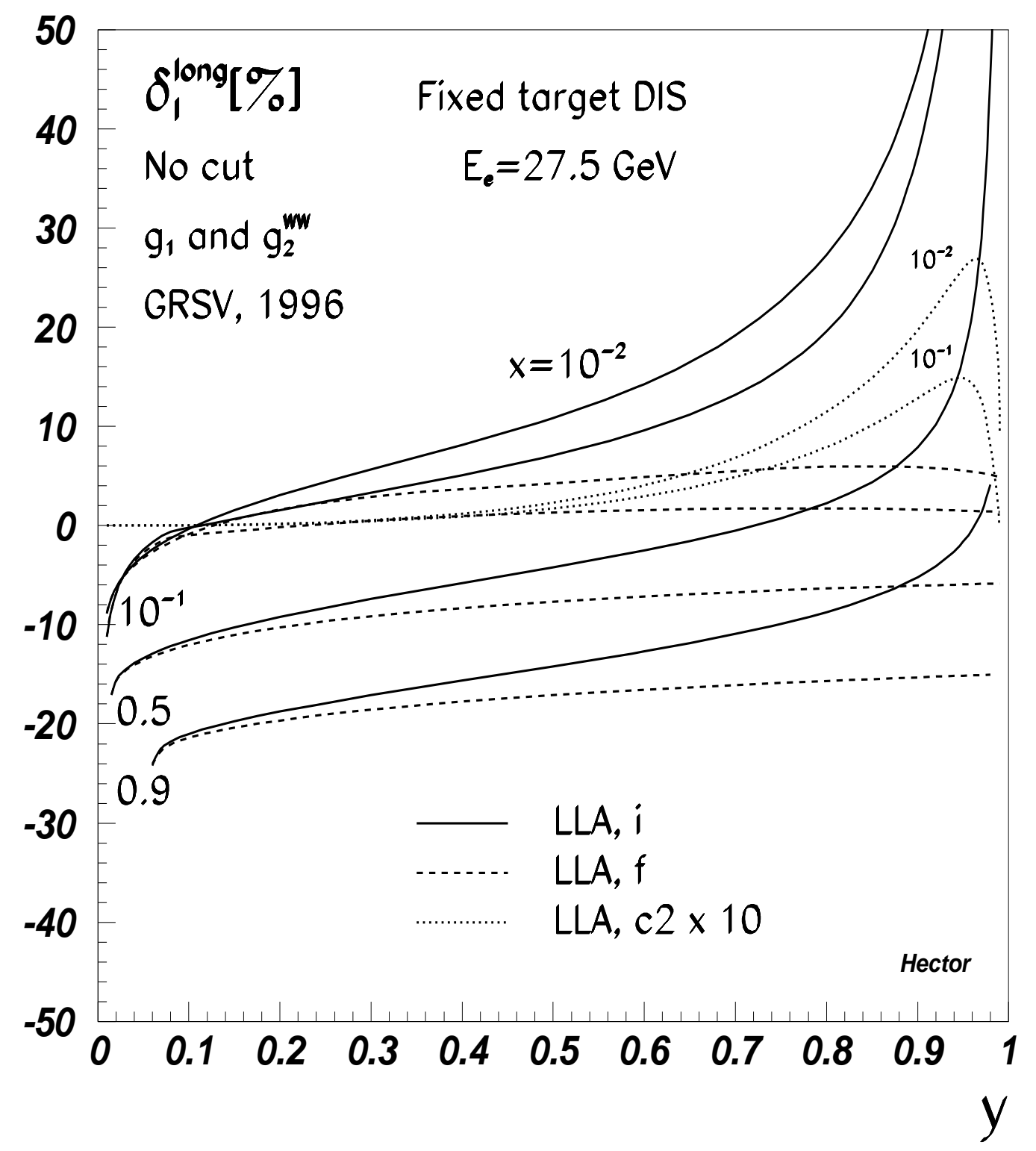

Figure 7 : Comparison of the different contributions to the $O(\alpha)$ leptonic QED corrections in LLA for longitudinally polarized protons at $\sqrt{S}=7.4 \mathrm{GeV}$. Full lines : initial state radiation; dashed lines: final state radiation; dotted lines: Compton contribution, eq. (101), scaled by a factor 10 . 


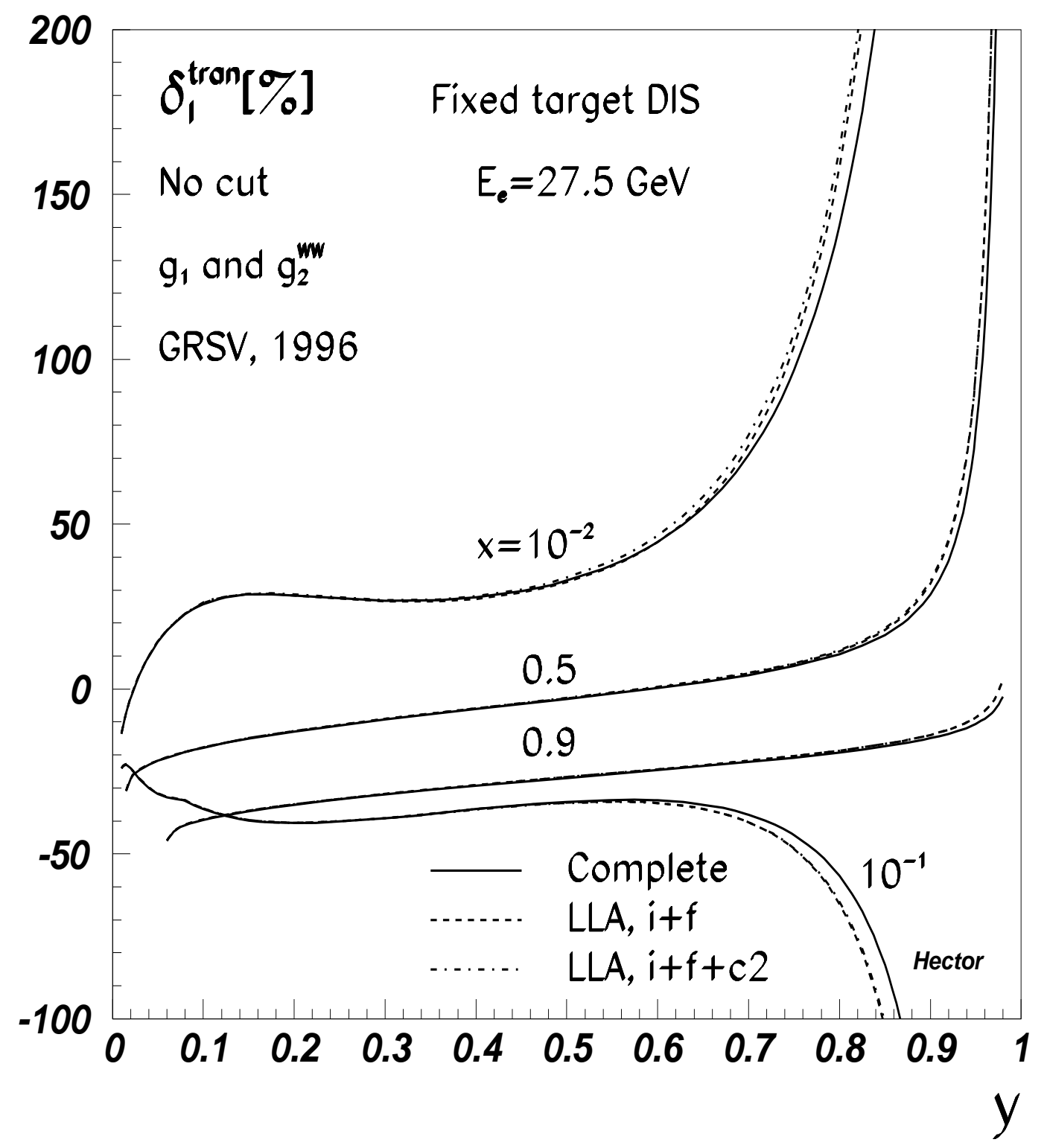

Figure 8: $O(\alpha)$ leptonic QED correction, eq. (47), to the polarized part of the differential deepinelastic scattering cross section for transversely polarized protons at $\sqrt{S}=7.4 \mathrm{GeV}$. Full lines : complete corrections; dashed lines : initial and final-state Bremsstrahlung contributions in LLA; dashdotted lines : complete LLA contributions, eq. (94). 


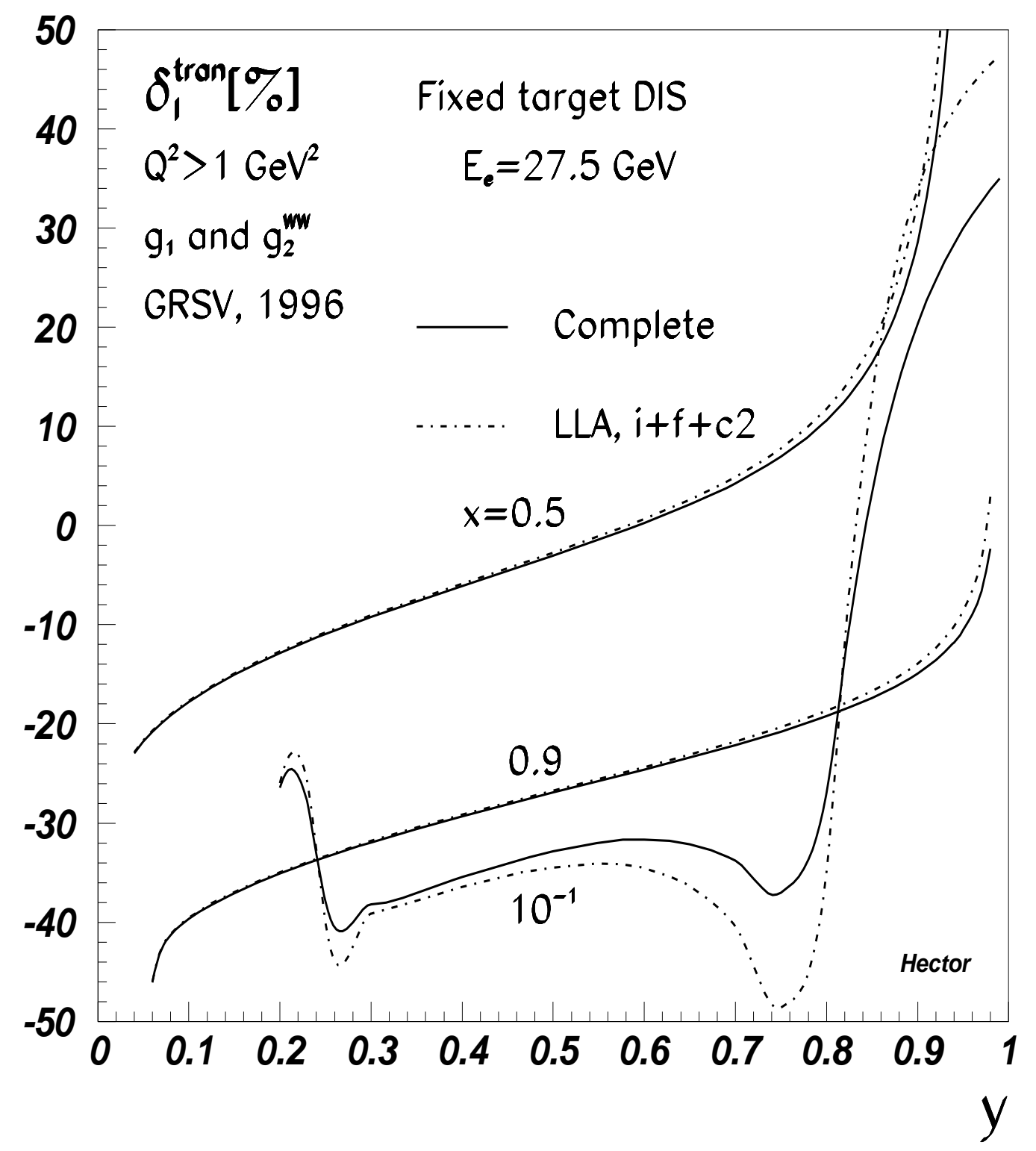

Figure 9: The same as in figure 8 applying a $Q^{2}$-cut of $Q_{h}^{2}>1 \mathrm{GeV}$. Full lines : complete corrections; dash-dotted lines : complete LLA corrections. 


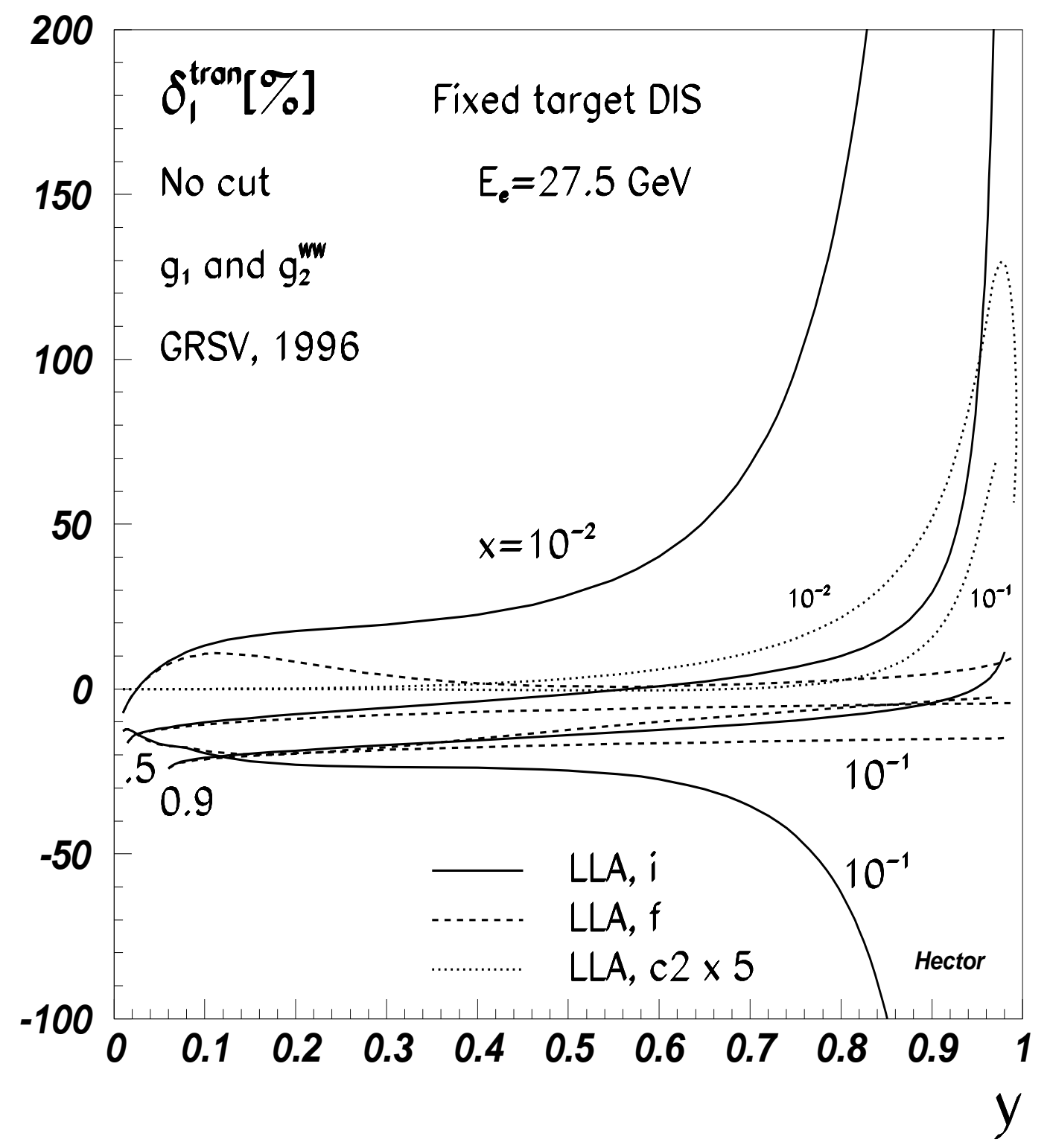

Figure 10 : Comparison of the different contributions to the $O(\alpha)$ leptonic QED corrections in LLA for transversely polarized protons at $\sqrt{S}=7.4 \mathrm{GeV}$. Full lines : initial state radiation; dashed lines: final state radiation; dotted lines: Compton contribution, eq. (101), scaled by a factor 5 . 


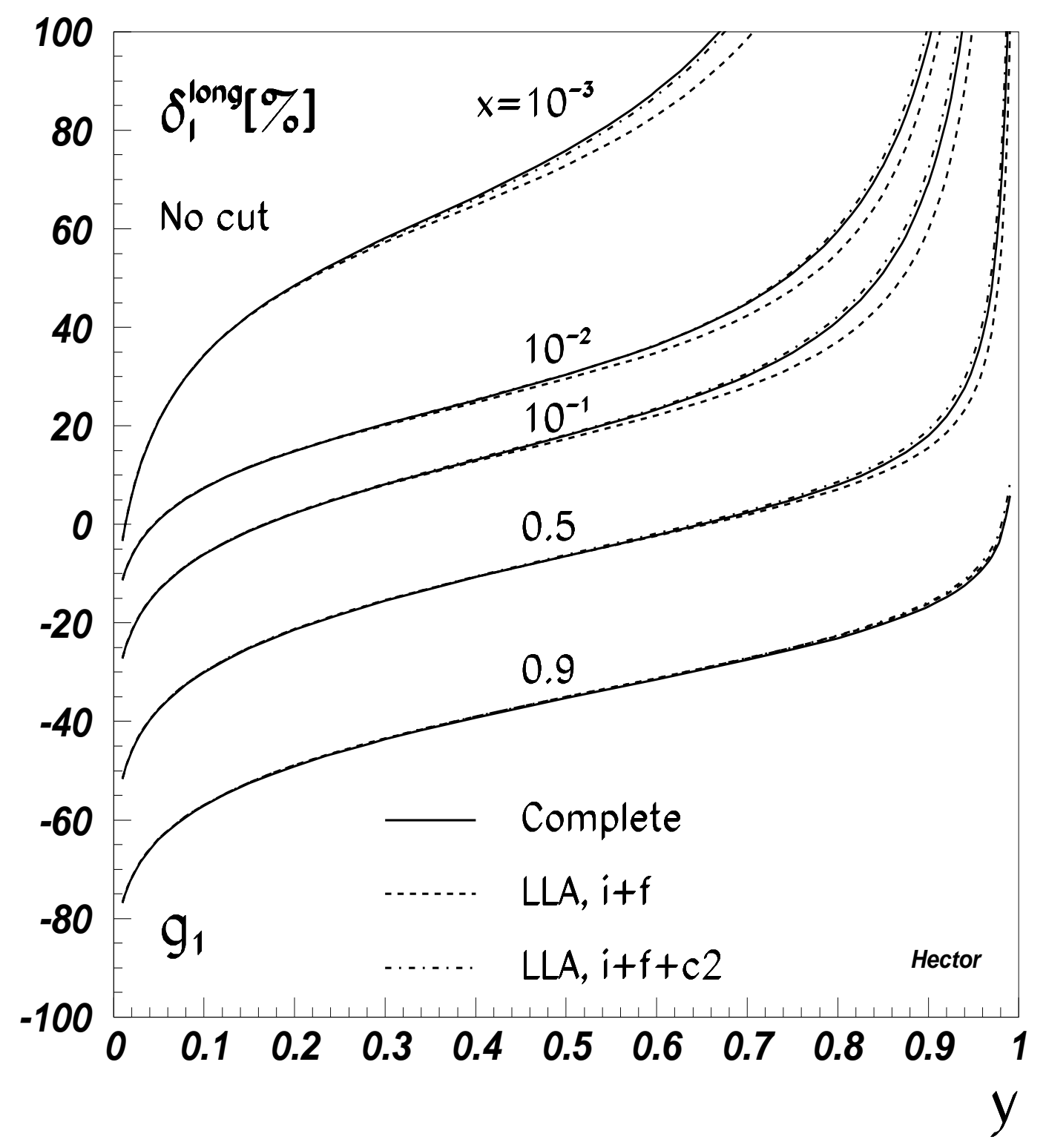

Figure 11: $O(\alpha)$ leptonic QED correction, eq. (47), to the polarized part of the differential deepinelastic scattering cross section for longitudinally polarized protons at $\sqrt{S}=314 \mathrm{GeV}$. Full lines : complete corrections; dashed lines: LLA terms, eq. (94). 


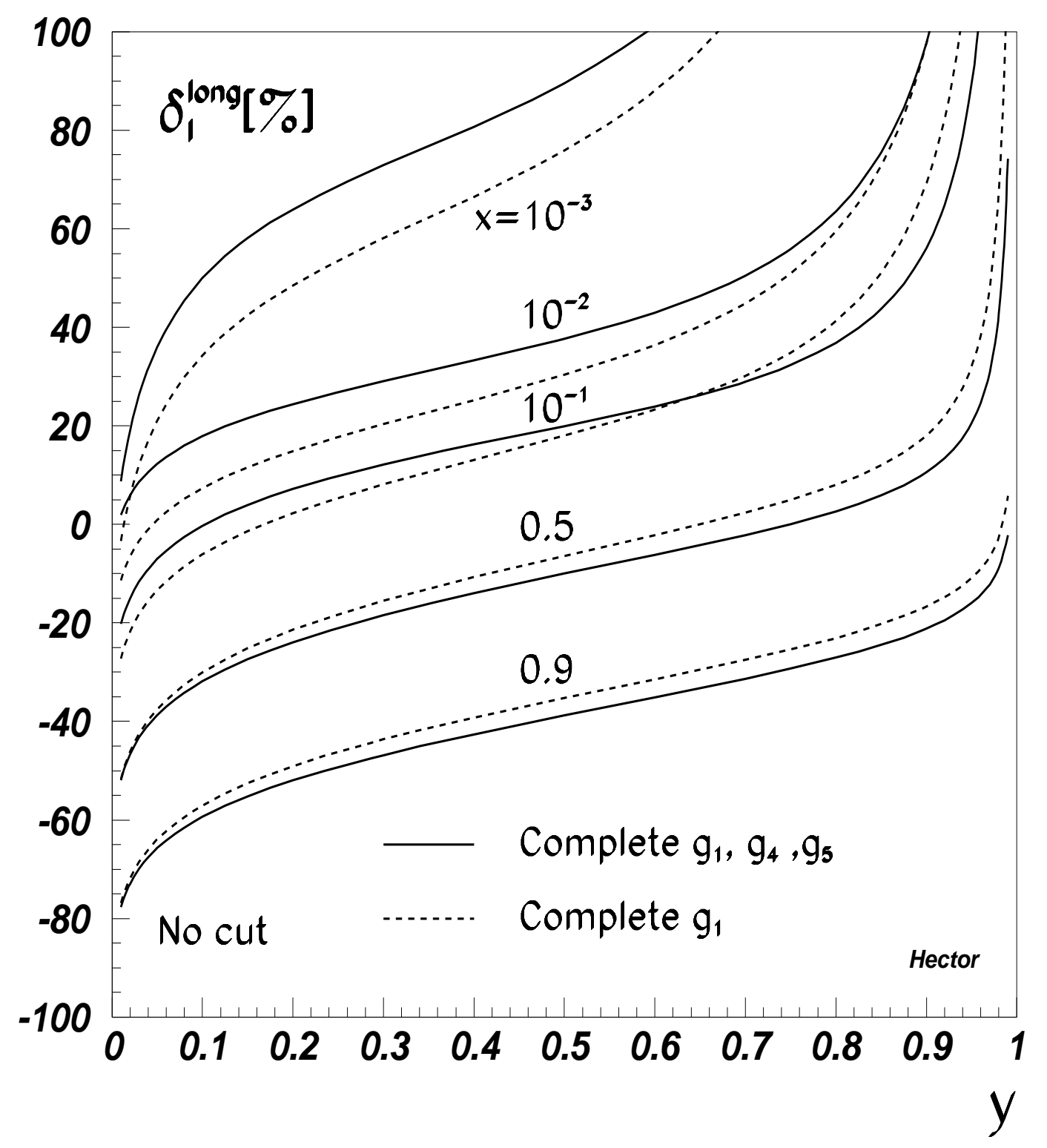

Figure 12: $O(\alpha)$ leptonic QED correction, eq. (47), to the polarized part of the differential deepinelastic scattering cross section for longitudinally polarized protons at $\sqrt{S}=314 \mathrm{GeV}$. Dashed lines : $\delta_{1}^{\text {long }}$ for only the structure function $g_{1}$; full lines : complete correction. The contributions due to the structure functions $g_{2}$ and $g_{3}$ are of $O\left(M^{2} / S\right)$ and are not included. 